\title{
SHARP STABILITY ESTIMATES FOR QUASI-AUTONOMOUS EVOLUTION EQUATIONS OF HYPERBOLIC TYPE
}

$\mathrm{BY}$

\author{
PHILIPPE SOUPLET
}

Laboratoire Analyse Géométrie et Applications, UMR CNRS 7539, Institut Galilée, Université Paris-Nord, 93430 Villetaneuse, France

Abstract. We study the energy decay of the difference of two solutions for dissipative evolution problems of the type:

$$
u^{\prime \prime}+L u+g\left(u^{\prime}\right)=h(t), \quad t \geq 0,
$$

including wave and plate equations and ordinary differential equations. In the general case, when the damping term $g$ behaves like a power of the velocity $u^{\prime}$, the energy decreases like a negative power of time, multiplied by a constant depending on the initial energies. We provide estimates on these constants and prove their optimality. In the special case of the ordinary differential equation with periodic forcing, we establish, relying on a controllability-like technique, that the decay is in fact exponential, even under very weak damping.

Résumé. On étudie la décroissance de l'énergie pour la différence de deux solutions dans des problèmes d'évolution dissipatifs du type:

$$
u^{\prime \prime}+L u+g\left(u^{\prime}\right)=h(t), \quad t \geq 0 .
$$

Ceci s'applique en particulier aux équations des ondes et des plaques et à des équations différentielles ordinaires. Dans le cas général, et lorsque le terme d'amortissement $g$ se comporte comme une puissance de la vélocité $u^{\prime}$, l'énergie décroît comme une puissance négative du temps, que multiplie une constante dépendant des énergies initiales. On donne des estimations sur ces constantes et on prouve leur optimalité. Dans le cas de l'équation différentielle ordinaire avec un terme source périodique, on montre, en utilisant une technique de type contrôlabilité, que la décroissance en temps est en fait exponentielle, et ce même en présence d'un amortissement très faible.

Received February 8, 1996.

1991 Mathematics Subject Classification. Primary 35L70, 35L75, 35B35, 35B40; Secondary 34D05.

E-mail address: souplet@math.univ-paris13.fr 
0. Introduction. This paper is devoted to the study of quasi-autonomous dissipative equations that can be collected in the following abstract form:

$$
u^{\prime \prime}+L u+g\left(u^{\prime}\right)=h(t), \quad t \in \mathbf{R}^{+}
$$

where $L$ is an (unbounded) selfadjoint linear operator, positive and coercive, on a real Hilbert space $H, g: \mathbf{R} \rightarrow \mathbf{R}$ is a generally nondecreasing continuous function, such that $g(0)=0$, and $h$ is an $H$-valued function. We shall deal with two kinds of equations:

- Partial differential equations of the form:

$$
\left\{\begin{array}{l}
u^{\prime \prime}+L u+g\left(u^{\prime}\right)=h(t, x) \quad \text { in } \mathbf{R}^{+} \times \Omega, \\
u \in C\left(\mathbf{R}^{+} ; V\right) \cap C^{1}\left(\mathbf{R}^{+} ; H\right)
\end{array}\right.
$$

with $\Omega$ a bounded domain in $\mathbf{R}^{N}, H=L^{2}(\Omega)$, and $V=D\left(L^{1 / 2}\right)$. The main examples are the wave equation:

$$
\begin{cases}u_{t t}-\Delta u+g\left(u_{t}\right)=h(t, x) & \text { in } \mathbf{R}^{+} \times \Omega \\ u=0, & \text { in } \mathbf{R}^{+} \times \partial \Omega\end{cases}
$$

corresponding to the choice $V=H_{0}^{1}(\Omega)$ and $L=-\Delta$, and the plate equation

$$
\begin{cases}u_{t t}+\Delta^{2} u+g\left(u_{t}\right)=h(t, x) & \text { in } \mathbf{R}^{+} \times \Omega, \\ u=\nabla u=0 & \text { in } \mathbf{R}^{+} \times \partial \Omega,\end{cases}
$$

with $V=H_{0}^{2}(\Omega)$ and $L=\Delta^{2}$.

- Ordinary differential equations (ODE) of the type

$$
u^{\prime \prime}+u+g\left(u^{\prime}\right)=h(t), \quad t \in \mathbf{R}^{+},
$$

corresponding to the case $H=V=\mathbf{R}, L=$ Id.

We shall here study the asymptotic stability of these equations, that is, the decay of the difference of two solutions to 0 as $t \rightarrow+\infty$.

The energy being defined for $(0.2)$ by

$$
E_{u}(t)=\frac{1}{2}\left(\left\|u^{\prime}\right\|_{H}^{2}+\|u\|_{V}^{2}\right),
$$

(resp. for $(0.5)$ by

$$
\left.E_{u}(t)=\frac{1}{2}\left(u^{2}+u^{\prime 2}\right)\right),
$$

we shall always assume in the sequel that $h$ is non-resonant, i.e., that the equation admits a solution $u$ such that $E_{u}$ is bounded on $\mathbf{R}^{+}$, and we know that, in this case, all solutions have a bounded energy. For details on the initial-value problem associated with (0.2), we refer to [6], [8] and the references therein.

We already know that, when $g$ essentially grows like a power greater than 1 in the neighbourhood of 0 , the difference of two solutions $u$ and $v$ converges to 0 in the energy space $H \times V$ as $t \rightarrow+\infty$, like a negative power of $t$ :

$$
E_{u-v}(t) \leq M\left(E_{u}(0), E_{v}(0)\right) t^{\frac{-2}{p-1}}
$$

where $M$ depends on the initial energies in a bounded way. On the other hand, in the special case when $h \equiv 0$ (homogeneous problem), sharp estimates on $M$ are derived in 
[3]. For instance, if $g\left(u^{\prime}\right)$ is of the type $\left|u^{\prime}\right|^{p-1} u^{\prime}+\left|u^{\prime}\right|^{q-1} u^{\prime}$, with $9 \geq p>1$, it is proved that

$$
E_{u}(t) \leq C\left(1+\left[E_{u}(0)\right]^{\frac{p q-1}{p(q-1)}}\right) t^{\frac{-2}{p-1}},
$$

for some constant $C>0$ independent of the initial data.

The aim of the first section is to provide precise information, in the general quasiautonomous case, on the behaviour, for large energies, of the constant $M$ that appears in (0.6). In order to do so, we shall resume and extend the methods of Haraux, Zuazua ([6], [8]), and Carpio ([3]), based on the use of adapted Liapunov functionals, obtained by perturbating the energy, leading to differential inequalities. For the wave and plate equations, in the nicer case when $p=q$, we extend to all $h$ the estimate established in [3] for $h \equiv 0$, an estimate that was proved to the optimal ([4]) when $g$ is a power.

The second section presents a generalization of a result of Haraux [7] concerning the stabilization of damped abstract evolution equations of the type

$$
y^{\prime \prime}+A y+B y^{\prime}=0,
$$

where $A$ is an unbounded selfadjoint operator, positive and coercive, and $B$ a positive selfadjoint bounded operator. That result claimed that the zero solution was exponentially stable, whenever the energy of the solutions of the conservative equation $\phi^{\prime \prime}+A \phi=0$ could be controlled or "observed") by means of the operator $B^{1 / 2}$ on a sufficiently long time interval.

Our contribution here is the extension of that property to the case of a non-constant nonlinear damping, a feature that will be useful in the sequel.

In the third section, we are looking for stronger time estimates in the case of the ODE $(0.5)$, when $h$ is non-constant periodic. The idea of such a possibility is suggested by the fact (cf. [1]) that for first-order equations of the type

$$
u^{\prime}+u^{3}=f(t),
$$

the difference of two solutions decays exponentially to zero when $f$ is non-constant periodic, while the decay is only polynomial when, for instance, $f=0$.

Our main result is the exponential stability of the periodic solution:

$$
E_{u-v}(t) \leq M\left(E_{u}(0), E_{v}(0)\right) e^{-\delta t} .
$$

When $g$ (nondecreasing) is of class $C^{1}$, this happens whenever $g \not \equiv 0$ in some neighbourhood of 0 and the condition is then optimal, for it is necessary for any type of stability. The result is proved via the observability property of section 2 , which applies here. Indeed, when writing the difference of two solutions to (0.5), one obtains an equation involving a non-constant linear dissipative term. Last, when $g$ satisfies some polynomial growth assumptions, we become able to provide a precise estimate on the behaviour of the constant $M\left(E_{u}(0), E_{v}(0)\right)$ involved in (0.7), with an exponential growth for large energies.

In section 4, we prove the optimality of the constants of sections 1 and 3 for the ODE, in the cases of both polynomial and exponential stabilities. In order to do so, we resume the technique introduced in [3]. It consists in taking profit of the existence of a special solution to (0.5), which is global on the whole real line, and whose energy grows like 
a power of $|t|$ as $t$ tends to $-\infty$, thus enabling us to investigate the behaviour of the constant in the whole range of energies. The existence of this global solution and its asymptotic properties are established by adapting the methods of [9], [10].

1. Polynomial decay estimates for hyperbolic problems in bounded domains. Let $\Omega$ be a bounded domain in $\mathbf{R}^{N}$ and $H=L^{2}(\Omega)$. Let $V$ be a real Hilbert space, such that $V \subset H$ with continuous and dense imbedding, and $L^{\infty}(\Omega) \cap V$ is dense in $V$. We denote by $|\cdot|$ and $\|\cdot\|$ the norms on $H$ and $V$ respectively and by $a(\cdot, \cdot)$ the inner product on $V$. For any function $u \in C\left(\mathbf{R}^{+} ; V\right) \cap C^{1}\left(\mathbf{R}^{+} ; H\right)$, we define the associated energy as

$$
E_{u}(t):=\frac{1}{2}\left(\left|u^{\prime}(t)\right|^{2}+\|u(t)\|^{2}\right) .
$$

Let $L \in \mathcal{L}\left(V, V^{\prime}\right)$ be the unique operator such that

$$
\forall u, v \in V, \quad\langle L u, v\rangle=a(u, v) .
$$

Let $g: \mathbf{R} \rightarrow \mathbf{R}$ be a nondecreasing continuous function such that $g(0)=0$ and $h \in$ $S^{1}\left(\mathbf{R}^{+} ; H\right)$, with

$$
S^{1}\left(\mathbf{R}^{+} ; H\right)=\left\{h \in L_{\mathrm{loc}}^{1}\left(\mathbf{R}^{+} ; H\right)\left|h^{*}:=\sup _{t \geq 0} \int_{t}^{t+1}\right| h(s) \mid d s<+\infty\right\} .
$$

We consider the abstract evolution equation

$$
\left\{\begin{array}{l}
u^{\prime \prime}+L u+g\left(u^{\prime}\right)=h \quad \text { in } \mathbf{R}^{+} \times \Omega, \\
u \in C\left(\mathbf{R}^{+} ; V\right) \cap C^{1}\left(\mathbf{R}^{+} ; H\right) .
\end{array}\right.
$$

Our more general result can be stated as follows:

ThEOREM 1.1. We assume that $g$ is continuous with $g(0)=0$ and satisfies either of the hypotheses (i) or (ii) below.

(i) There exists $q>1$ such that

$$
V \subset L^{q+1}(\Omega) \text { with continuous imbedding, }
$$

there exists $p$ with $1<p \leq q$ and $c, K>0$ such that

$$
\begin{gathered}
\forall x, y \in \mathbf{R}, \quad c|x-y|^{p+1} \leq(g(x)-g(y))(x-y), \\
\forall x, y \in \mathbf{R}, \quad|g(x)-g(y)| \leq K\left[1+(g(x) x+g(y) y)^{\frac{q-1}{q+1}}\right]|x-y| .
\end{gathered}
$$

(ii) We have

$$
V \subset L^{\infty}(\Omega) \text { with continuous imbedding, }
$$

and there exists $p>1$ and $c, K>0$ such that (1.6) holds and

$$
\forall x, y \in \mathbf{R}, \quad|g(x)-g(y)| \leq K[1+g(x) x+g(y) y]|x-y| .
$$

Then, whenever $h \in S^{1}\left(\mathbf{R}^{+} ; H\right)$ is non-resonant, any pair of solutions $u, v$ of (1.4) satisfies

$$
\forall t \geq 0, \quad E_{u-v}(t) \leq\left(F\left(E_{u}(0)\right)+F\left(E_{v}(0)\right)\right) t^{\frac{-2}{p-1}},
$$


with, in case (i),

$$
F(X)=C\left(1+X^{1+\frac{2(q-1)}{(q+1)(p-1)}}\right)
$$

in case (ii),

$$
F(X)=C\left(1+X^{1+\frac{2}{p-1}}\right)
$$

and $C>0$ independent of $u$ and $v$.

Proof. Let $u$ be a fixed bounded solution of (1.4) and $v$ another solution. It obviously suffices to prove that

$$
E_{u-v}(t) \leq F\left(E_{u-v}(0)\right) t^{\frac{-2}{p-1}}
$$

where the constant $C$ involved in the expression of $F$ possibly depends on $u$ (but not on $v)$. Indeed, if $v_{1}$ and $v_{2}$ are any solutions of (1.4), we shall then have

$$
\begin{aligned}
E_{v_{1}-v_{2}}(t) & \leq 2 E_{v_{1}-u}(t)+2 E_{u-v_{2}}(t) \\
& \leq 2\left[F\left(E_{v_{1}-u}(0)\right)+F\left(E_{u-v_{2}}(0)\right)\right] t^{\frac{-2}{p-1}} \\
& \leq 2\left[F\left(2 E_{v_{1}}(0)+2 E_{u}(0)\right)+F\left(2 E_{v_{2}}(0)+2 E_{u}(0)\right)\right] t^{\frac{-2}{p-1}} \\
& \leq C^{\prime}\left[F\left(E_{u}(0)\right)+F\left(E_{v_{1}}(0)\right)+F\left(E_{v_{2}}(0)\right)\right] t^{\frac{-2}{p-1}}
\end{aligned}
$$

for some $C^{\prime}>0$ (depending only on $p$ and $q$ ), which immediately yields the result.

On the other hand, we know that we may assume $u(0), v(0) \in D(A), u^{\prime}(0), v^{\prime}(0) \in V$, $g\left(u^{\prime}(0)\right), g\left(v^{\prime}(0)\right) \in H$, and $h \in C^{1}\left(\mathbf{R}^{+} ; H\right)$, so that

$$
u \in W_{\mathrm{loc}}^{2, \infty}\left(\mathbf{R}^{+} ; H\right) \cap W_{\mathrm{loc}}^{1, \infty}\left(\mathbf{R}^{+} ; V\right) \text {. }
$$

The result will then extend to all weak solutions by a standard density argument relying on the fact that $F$ depends on the initial energies in a bounded way.

We denote $w=u-v, E=E_{w}$ and we set

$$
\phi(t)=(1+k \varepsilon) E(t)+\varepsilon E^{\frac{p-1}{2}}\left(w, w^{\prime}\right)
$$

with $\varepsilon, k>0$ to be determined later. In the sequel, all the constants $C_{i}>0$ will possibly depend on $u$, but not on $v$. From (1.13), we know that $E$ and $\left(w, w^{\prime}\right)$ are in $W_{\text {loc }}^{1, \infty}\left(\mathbf{R}^{+}\right)$ and for a.e. $t \geq 0$ :

$$
\begin{aligned}
E^{\prime}(t) & =-\left(g\left(u^{\prime}\right)-g\left(v^{\prime}\right), u^{\prime}-v^{\prime}\right) \leq 0, \\
\left(w, w^{\prime}\right)(t) & =\left|w^{\prime}\right|^{2}-\|w\|^{2}-\left(g\left(u^{\prime}\right)-g\left(v^{\prime}\right), w\right) .
\end{aligned}
$$

For a.e. $t \geq 0$, we have (the variable $t$ being omitted for convenience):

$$
\phi^{\prime}=(1+k \varepsilon) E^{\prime}+\varepsilon \frac{p-1}{2} E^{\frac{p-3}{2}}\left(w, w^{\prime}\right) E^{\prime}+\varepsilon E^{\frac{p-1}{2}}\left(\left|w^{\prime}\right|^{2}-\|w\|^{2}-\left(g\left(u^{\prime}\right)-g\left(v^{\prime}\right), w\right)\right) .
$$

By the Cauchy-Schwarz inequality and the continuous imbedding $V \subset H$, we have

$$
\left|\left(w, w^{\prime}\right)\right| \leq|w|\left|w^{\prime}\right| \leq C_{1}\|w\|\left|w^{\prime}\right| \leq C_{1} E
$$


hence, since $E$ is nonincreasing:

$$
\phi^{\prime} \leq\left(1+k \varepsilon-\varepsilon \frac{p-1}{2} C_{1} E(0)^{\frac{p-1}{2}}\right) E^{\prime}+\varepsilon E^{\frac{p-1}{2}}\left(\left|w^{\prime}\right|^{2}-\|w\|^{2}+\left|\left(g\left(u^{\prime}\right)-g\left(v^{\prime}\right), w\right)\right|\right) .
$$

Choosing $k=\frac{p-1}{2} C_{1} E(0)^{\frac{p-1}{2}}$, this becomes

$$
\begin{gathered}
\phi^{\prime} \leq \overbrace{-\left(g\left(u^{\prime}\right)-g\left(v^{\prime}\right), u^{\prime}-v^{\prime}\right)}^{\mathrm{T} 1} \overbrace{-2 \varepsilon E^{\frac{p+1}{2}}}^{\mathrm{T} 2} \overbrace{+2 \varepsilon E^{\frac{p-1}{2}}\left|w^{\prime}\right|^{2}}^{\mathrm{T} 3} \\
\underbrace{+\varepsilon E^{\frac{p-1}{2}}\left|\left(g\left(u^{\prime}\right)-g\left(v^{\prime}\right), w\right)\right|}_{\mathrm{T} 4}
\end{gathered}
$$

and, from (1.16) and the nonincreasing character of $E$, we see that

$$
\frac{1}{2} E \leq \phi \leq 2 E
$$

provided that

$$
\varepsilon \leq \varepsilon_{0}:=\frac{C_{2}}{1+E(0)^{\frac{p-1}{2}}} .
$$

First, using Young's inequality with exponents $(p+1) /(p-1)$ and $(p+1) / 2$ and the fact that $p+1 \geq 2$ and $\Omega$ is bounded, we get

$$
\begin{aligned}
\mathrm{T} 3 & \leq \varepsilon\left(E^{\frac{p+1}{2}}+C_{3}\left|w^{\prime}\right|^{p+1}\right) \\
& \leq \varepsilon\left(E^{\frac{p+1}{2}}+C_{4}\left\|w^{\prime}\right\|_{p+1}^{p+1}\right) .
\end{aligned}
$$

Dealing with the term T4 in formula (1.17), we can write for any $\tau>0$ :

$$
\begin{aligned}
\left|\left(g\left(u^{\prime}\right)-g\left(v^{\prime}\right), w\right)\right| & \leq \int_{\Omega} \frac{g\left(u^{\prime}\right)-g\left(v^{\prime}\right)}{u^{\prime}-v^{\prime}}|w|\left|w^{\prime}\right| d x \\
& \leq \int_{\Omega} \frac{g\left(u^{\prime}\right)-g\left(v^{\prime}\right)}{u^{\prime}-v^{\prime}}\left[\tau|w|^{2}+\frac{1}{4 \tau}\left|w^{\prime}\right|^{2}\right] d x .
\end{aligned}
$$

CASE (I). From (1.7), Hölder's inequality applied with exponents $(q+1) /(q-1)$ and $(q+1) / 2$, and the continuous imbedding $V \subset L^{q+1}(\Omega)$, we have, for any $\tau>0$ :

$$
\begin{aligned}
\left|\left(g\left(u^{\prime}\right)-g\left(v^{\prime}\right), w\right)\right| & \leq K \tau \int_{\Omega}\left[1+\left(g\left(u^{\prime}\right) u^{\prime}+g\left(v^{\prime}\right) v^{\prime}\right)^{\frac{q-1}{q+1}}|w|^{2}\right. \\
& \leq \tau f(t)\|w\|^{2}+\frac{1}{4 \tau} \int_{\Omega}\left(g\left(u^{\prime}\right)-g\left(v^{\prime}\right)\right) w^{\prime} d x
\end{aligned}
$$

with

$$
f(t)=C_{5}\left\{1+\left[\int_{\Omega}\left(g\left(u^{\prime}\right) u^{\prime}+g\left(v^{\prime}\right) v^{\prime}\right) d x\right]^{\frac{q-1}{q+1}}\right\}
$$

and the term $\mathrm{T} 4$ can be estimated as

$$
\mathrm{T} 4 \leq \varepsilon\left(2 \tau f(t) E^{\frac{p+1}{2}}+\frac{E(0)^{\frac{p-1}{2}}}{4 \tau}\left(g\left(u^{\prime}\right)-g\left(v^{\prime}\right), w^{\prime}\right)\right) .
$$


On the other hand, we know (cf. [8], Theorem 1.1) that every solution $v$ satisfies

$$
\begin{aligned}
\sup _{t \geq 0} \int_{t}^{t+1} \int_{\Omega} g\left(v^{\prime}\right) v^{\prime} d x d s & \leq \sup _{t \geq 0} E_{v}(t)+\sqrt{2} h^{*} \sup _{t \geq 0}\left[E_{v}(t)\right]^{1 / 2} \\
& \leq C_{6}\left(1+\sup _{t \geq 0} E_{v}(t)\right)
\end{aligned}
$$

so that

$$
\begin{aligned}
\sup _{t \geq 0} \int_{t}^{t+1} \int_{\Omega}\left(g\left(u^{\prime}\right) u^{\prime}+g\left(v^{\prime}\right) v^{\prime}\right) d x d s & \leq C_{6}\left(2+\sup _{t \geq 0} E_{u}(t)+\sup _{t \geq 0} E_{v}(t)\right) \\
& \leq C_{6}\left(2+3 \sup _{t \geq 0} E_{u}(t)+2 \sup _{t \geq 0} E_{w}(t)\right) \\
& \leq C_{7}\left(1+E_{w}(0)\right)
\end{aligned}
$$

and we get

$$
\sup _{t \geq 0} \int_{t}^{t+1} f(s) d s \leq C_{8}\left(1+E_{w}(0)^{\frac{q-1}{q+1}}\right) .
$$

By putting inequalities (1.17), (1.20), (1.21) together, we obtain

$$
\begin{aligned}
\phi^{\prime} \leq & -\left(g\left(u^{\prime}\right)-g\left(v^{\prime}\right), u^{\prime}-v^{\prime}\right)-2 \varepsilon E^{\frac{p+1}{2}}+\varepsilon E^{\frac{p+1}{2}}+C_{4} \varepsilon\left\|w^{\prime}\right\|_{p+1}^{p+1} \\
& +2 \varepsilon \tau f(t) E^{\frac{p+1}{2}}+\frac{\varepsilon E(0)^{\frac{p-1}{2}}}{4 \tau}\left(g\left(u^{\prime}\right)-g\left(v^{\prime}\right), w^{\prime}\right)
\end{aligned}
$$

and by rearranging the terms and using (1.6),

$$
\phi^{\prime} \leq\left(\frac{C_{4} \varepsilon}{c}+\frac{\varepsilon E(0)^{\frac{p-1}{2}}}{4 \tau}-1\right)\left(g\left(u^{\prime}\right)-g\left(v^{\prime}\right), u^{\prime}-v^{\prime}\right)+\varepsilon(2 \tau f(t)-1) E^{\frac{p+1}{2}} .
$$

Now, if we take in formula (1.24)

$$
\tau=\frac{C_{9}}{1+E(0)^{\frac{q-1}{q+1}}}
$$

and

$$
\varepsilon \leq \varepsilon_{1}:=\frac{C_{10}}{1+E(0)^{\frac{p-1}{2}+\frac{q-1}{q+1}}} \leq \varepsilon_{0},
$$

we find, thanks to (1.18) and (1.23):

$$
\phi^{\prime} \leq \varepsilon\left(m(t)-\frac{1}{2}\right) E^{\frac{p+1}{2}} \leq C_{11} \varepsilon\left(m(t)-\frac{1}{2}\right) \phi^{\frac{p+1}{2}}
$$

with $m$ satisfying

$$
\sup _{t \geq 0} \int_{t}^{t+1} m(s) d s \leq \frac{1}{4}
$$

At this point, we shall use the following simple Lemma that we recall without proof (cf. [8], Lemma 1.7): 
LEMma 1.2. Let $\phi \in W_{\text {loc }}^{1,1}\left(\mathbf{R}^{+} ; \mathbf{R}\right)$ and $f \in L_{\text {loc }}^{1}\left(\mathbf{R}^{+} ; \mathbf{R}\right)$ be two nonnegative functions such that

$$
\begin{gathered}
f^{*}:=\sup _{t \geq 0} \int_{t}^{t+1} f(t) d t<\eta, \\
\phi^{\prime}(t) \leq(f(t)-\eta)[\phi(t)]^{a} \quad \text { a.e. on } \mathbf{R}^{+}
\end{gathered}
$$

with $a>1$. Then

$$
\phi(t) \leq M t^{\frac{-1}{p-1}} \quad \text { for all } t \geq T_{0}:=\frac{2 f^{*}}{\eta-f^{*}}
$$

with

$$
M=\left[\frac{\left(\eta-f^{*}\right)(a-1)}{2}\right]^{\frac{-1}{a-1}} .
$$

Applying Lemma 1.2 and choosing $\varepsilon=\varepsilon_{1}$, we finally obtain, for $t \geq T_{0}=2$,

$$
E(t) \leq 2 \phi(t) \leq C\left[1+E(0)^{1+\frac{2(q-1)}{(q+1)(p-1)}}\right] t^{-\frac{2}{p-1}},
$$

and since $E \leq E(0)$ the result finally extends to all $t \geq 0$.

CASE (II). The proof is exactly similar, provided one is taking

$$
\begin{gathered}
f(t)=K\left\{1+\int_{\Omega}\left(g\left(u^{\prime}\right) u^{\prime}+g\left(v^{\prime}\right) v^{\prime}\right) d x\right\}, \\
\tau=\frac{C_{9}}{1+E(0)}
\end{gathered}
$$

and

$$
\varepsilon_{1}=\frac{C_{10}}{1+E(0)^{1+\frac{p-1}{2}}}
$$

then yielding

$$
E(t) \leq C\left[1+E(0)^{1+\frac{2}{p-1}}\right] t^{-\frac{2}{p-1}}
$$

The proof is now complete.

The next result improves the estimate of Theorem 1.1 when $p=q$. In this case, we recover for general $h$ the same exponent in function $F$ as the one found when $h \equiv 0$ (cf. [3]). It is therefore interesting to recall that, in the special case of the homogeneous wave equation with $g\left(u^{\prime}\right)=\left|u^{\prime}\right|^{p-1} u^{\prime}$, the growth of $F$ is known to be optimal ([4]).

TheOREM 1.3. We assume that $g$ is continuous with $g(0)=0$ and satisfies (i) in Theorem 1.1 with $p=q$. Then, whenever $h \in S^{1}\left(\mathbf{R}^{+} ; H\right)$ is non-resonant, any pair of solutions $u$, $v$ of (1.4) satisfies (1.10) with

$$
F(X)=C\left(1+X^{1+\frac{1}{p}}\right)
$$

and $C>0$ independent of $u$ and $v$. 
Proof. We are resuming the proof of Theorem 1.1 after inequality (1.20), the beginning being identical. The only difference then lies in the treatment of the term T4. We first note that assumption (1.7) implies, in particular,

$$
\forall x \in \mathbf{R}, \quad g(x) x \leq C_{5}\left(1+|x|^{q+1}\right)
$$

(indeed, by taking $y=0$ in (1.7), we get, for any real $x$, either $g(x) x \leq 1$ or $|g(x)| \leq$ $2 K(g(x) x)^{(q-1) /(q+1)}|x|$, that is, $\left.g(x) x \leq(2 K)^{(q+1) / 2}|x|^{q+1}\right)$. Taking now $p=q$, we have, a.e. on $\mathbf{R}^{+} \times \Omega$ :

$$
\begin{aligned}
\left|g\left(u^{\prime}\right)-g\left(v^{\prime}\right)\right| & \leq C_{6}\left(1+\left|u^{\prime}\right|^{p-1}+\left|v^{\prime}\right|^{p-1}\right)\left|w^{\prime}\right| \\
& \leq C_{7}\left(1+\left|u^{\prime}\right|^{p-1}+\left|w^{\prime}\right|^{p-1}\right)\left|w^{\prime}\right|
\end{aligned}
$$

and, by repeatedly applying Hölder's inequality, we get:

$$
\begin{aligned}
\left|\left(g\left(u^{\prime}\right)-g\left(v^{\prime}\right), w\right)\right| \leq & C_{7} \int_{\Omega}\left\{\left(1+\left|u^{\prime}\right|^{p-1}\right)\left|w^{\prime}\right|+\left|w^{\prime}\right|^{p}\right\}|w| d x \\
\leq & C_{8}\left(1+\int_{\Omega}\left|u^{\prime}\right|^{p+1} d x\right)^{\frac{p-1}{p+1}}\left(\int_{\Omega}\left(\left|w^{\prime}\right||w|\right)^{\frac{p+1}{2}} d x\right)^{\frac{2}{p+1}} \\
& \quad+C_{7}\left(\int_{\Omega}\left|w^{\prime}\right|^{p+1} d x\right)^{\frac{p}{p+1}}\left(\int_{\Omega}|w|^{p+1} d x\right)^{\frac{1}{p+1}} \\
\leq & C_{8}\left(1+\left\|u^{\prime}\right\|_{p+1}^{p-1}\right)\left\|w^{\prime}\right\|_{p+1}\|w\|_{p+1}+C_{7}\left\|w^{\prime}\right\|_{p+1}^{p}\|w\|_{p+1} .
\end{aligned}
$$

From (1.6) and the continuous imbedding $V \subset L^{p+1}(\Omega)$, we deduce

$$
\left|\left(g\left(u^{\prime}\right)-g\left(v^{\prime}\right), w\right)\right| \leq C_{9}\left(\left\|w^{\prime}\right\|_{p+1}^{p}+f(t)\left\|w^{\prime}\right\|_{p+1}\right)\|w\|
$$

with

$$
f(t)=1+\left(\int_{\Omega} g\left(u^{\prime}\right) u^{\prime} d x\right)^{\frac{p-1}{p+1}}
$$

hence

$$
\mathrm{T} 4 \leq \underbrace{C_{10} \varepsilon E^{\frac{p}{2}}\left\|w^{\prime}\right\|_{p+1}^{p}}_{\text {T5 }}+\underbrace{C_{10} \varepsilon f(t) E^{\frac{p}{2}}\left\|w^{\prime}\right\|_{p+1}}_{\text {T6 }}
$$

Let us now decompose the term T5. (We here keep two distinct exponents $p$ and $q$, for the calculation will be useful in the next theorem.)

$$
\mathrm{T} 5=C_{10} \varepsilon E^{\frac{p}{2}}\left\|w^{\prime}\right\|_{q+1}^{q} \leq C_{10}\left(\varepsilon^{\alpha} E(0)^{\beta \frac{p q-1}{2(q+1)}} E^{\frac{p+1}{2(q+1)}}\left(\varepsilon^{1-\alpha}\left\|w^{\prime}\right\|_{q+1}^{q} E(0)^{(1-\beta) \frac{p q-1}{2(q+1)}}\right),\right.
$$

where $0 \leq \alpha, \beta \leq 1$ are to be determined later. Applying Young's inequality with exponents $(q+1) / q$ and $q+1$ yields

$$
\mathrm{T} 5 \leq C_{11}\left(\varepsilon^{\alpha(q+1)} E(0)^{\beta \frac{p q-1}{2}} E^{\frac{p+1}{2}}+\varepsilon^{(1-\alpha)(q+1) / q}\left\|w^{\prime}\right\|_{q+1}^{q+1} E(0)^{(1-\beta) \frac{p q-1}{2 q}}\right) .
$$

Choosing $\alpha=(q+2) /(2(q+1))$ and $\beta=1 / 2$, this becomes

$$
\mathrm{T} 5 \leq C_{11}\left(\varepsilon \varepsilon^{q / 2} E(0)^{\frac{p q-1}{4}} E^{\frac{p+1}{2}}+\varepsilon^{1 / 2} E(0)^{\frac{p q-1}{4 q}}\left\|w^{\prime}\right\|_{q+1}^{q+1}\right) .
$$


Taking now $p=q$ again and applying Young's inequality with exponents $(p+1) / p$ and $p+1$ in the term T6, we obtain for any $\delta>0$ :

$$
\mathrm{T} 6 \leq C_{12} \varepsilon \delta^{\frac{1}{p}}[f(t)]^{\frac{p+1}{p}} E^{\frac{p+1}{2}}+\frac{\varepsilon}{\delta}\left\|w^{\prime}\right\|_{p+1}^{p+1} .
$$

Besides, we have, by (1.22)

$$
\sup _{t \geq 0} \int_{t}^{t+1}[f(t)]^{\frac{p+1}{p}} d t \leq C_{13}\left\{1+\sup _{t \geq 0}\left(\int_{t}^{t+1} \int_{\Omega} g\left(u^{\prime}\right) u^{\prime} d x d s\right)^{\frac{p-1}{p}}\right\}<+\infty
$$

and it is thus possible to choose $\delta$ small enough, independent of $v$, so that

$$
\mathrm{T} 6 \leq \varepsilon m(t) E^{\frac{p+1}{2}}+C_{14} \varepsilon\left\|w^{\prime}\right\|_{p+1}^{p+1},
$$

with $m$ satisfying

$$
\sup _{t \geq 0} \int_{t}^{t+1} m(s) d s \leq \frac{1}{4} .
$$

By putting inequalities (1.17), (1.20), (1.28), (1.29) with $p=q$, and (1.30) together, we obtain

$$
\begin{aligned}
\phi^{\prime} \leq & -\left(g\left(u^{\prime}\right)-g\left(v^{\prime}\right), u^{\prime}-v^{\prime}\right)-2 \varepsilon E^{\frac{p+1}{2}}+\varepsilon E^{\frac{p+1}{2}}+C_{4} \varepsilon\left\|w^{\prime}\right\|_{p+1}^{p+1} \\
& +C_{11}\left(\varepsilon \varepsilon^{p / 2} E(0)^{\frac{p^{2}-1}{4}} E^{\frac{p+1}{2}}+\varepsilon^{1 / 2} E(0)^{\frac{p^{2}-1}{4 p}}\left\|w^{\prime}\right\|_{p+1}^{p+1}\right)+\varepsilon m(t) E^{\frac{p+1}{2}}+C_{14} \varepsilon\left\|w^{\prime}\right\|_{p+1}^{p+1} .
\end{aligned}
$$

Hence, by using (1.6) and rearranging the terms:

$$
\begin{aligned}
\phi^{\prime} \leq & \varepsilon\left(-1+C_{11} \varepsilon^{p / 2} E(0)^{\frac{p^{2}-1}{4}}+m(t)\right) E^{\frac{p+1}{2}} \\
& +\left(-1+\frac{1}{c}\left(C_{4} \varepsilon+C_{11} \varepsilon^{1 / 2} E(0)^{\frac{p^{2}-1}{4 p}}+C_{14} \varepsilon\right)\right)\left(g\left(u^{\prime}\right)-g\left(v^{\prime}\right), u^{\prime}-v^{\prime}\right) .
\end{aligned}
$$

Now, if we take in formula (1.31)

$$
\varepsilon \leq \varepsilon_{1}:=\frac{C_{15}}{1+E(0)^{\frac{p^{2}-1}{2 p}}} \leq \varepsilon_{0}
$$

(with $\varepsilon_{0}$ as defined by $(1.19)$ ), we find

$$
\phi^{\prime} \leq \varepsilon\left(m(t)-\frac{1}{2}\right) E^{\frac{p+1}{2}} \leq-C_{16} \varepsilon\left(m(t)-\frac{1}{2}\right) \phi^{\frac{p+1}{2}} .
$$

We then conclude, as in Theorem 1.1, by applying Lemma 1.2 and choosing $\varepsilon=\varepsilon_{1}$.

REMARK 1.1. We recall that hypothesis (1.7) in Theorems $1.1,1.3$ is for instance satisfied if

$$
\begin{gathered}
\forall x, y \in \mathbf{R}, \quad|g(x)-g(y)| \leq K\left[1+|x|^{q-1}+|y|^{q-1}\right]|x-y|, \\
\forall x \in \mathbf{R}, \quad|g(x)| \geq c_{1}|x|^{q}-c_{2},
\end{gathered}
$$

and that hypothesis (1.9) is satisfied whenever, for $|x|$ large enough, $\rho:=\log |g|$ is of class $C_{1}$ and $\left|\rho^{\prime}(x)\right| \leq A|x|, A>0$. 
Concerning conditions (1.5) and (1.8) in the case of the wave equation (0.3), it is well known that $V \subset L^{r}(\Omega)$ with continuous imbedding when

$$
\begin{cases}r \in[1,+\infty], & \text { if } N=1, \\ r \in[1,+\infty), & \text { if } N=2, \\ r \in[1,2 N /(N-2)], & \text { if } N \geq 3 .\end{cases}
$$

In the case of ordinary differential equations, the improved method used in Theorem 1.3 works also for $q>p$, provided we assume the slightly reinforced growth condition (1.32) on $g$ for large values. The estimate is already known when $h \equiv 0$ and is optimal in this case (cf. [3]). The result can be stated as follows:

THEOREM 1.4 (Ordinary differential equations). We assume that $g$ is continuous with $g(0)=0$ and satisfies

$$
\begin{aligned}
& \forall x, y \in \mathbf{R}, \quad c\left(|x-y|^{p+1}+|x-y|^{q+1}\right) \leq(g(x)-g(y))(x-y), \\
& \forall x, y \in \mathbf{R}, \quad|g(x)-g(y)| \leq K\left[1+(g(x) x+g(y) y)^{\frac{q-1}{q+1}}\right]|x-y|
\end{aligned}
$$

for some $q \geq p>1$ and $c, K>0$. Then, whenever $h \in S^{1}\left(\mathbf{R}^{+}\right), h$ is non-resonant and any solutions $u, v$ of (0.5) satisfy (1.10) with

$$
F(X)=C\left(1+X^{\frac{p q-1}{p(q-1)}}\right)
$$

and $C>0$ independent of $u$ and $v$.

Proof. The non-resonance in the case of the ODE is known. It can be proved by the same technique as in [6], Theorem IV.2.1.1, so that we are just concerned with the decay estimate. The situation is quite simpler, since no infinite-dimensional functional space nor non-equivalent norms are involved. In fact, the proof follows exactly the same steps as in Theorem 1.3, up to replacing the inner products in $H$ by products of real numbers, all the norms by absolute values, and the energy by

$$
E_{u}(t):=\frac{1}{2}\left(u^{\prime 2}(t)+u^{2}(t)\right) .
$$

Once these changes have been adopted, we handle the term T4 in the following way. From (1.27), we get for any $t \geq 0$ :

$$
\left|g\left(u^{\prime}\right)-g\left(v^{\prime}\right)\right| \leq C_{6}\left(1+\left|u^{\prime}\right|^{q-1}+\left|w^{\prime}\right|^{q-1}\right)\left|w^{\prime}\right|,
$$

and, since $u$ is bounded on $\mathbf{R}^{+}$,

$$
\left|g\left(u^{\prime}\right)-g\left(v^{\prime}\right)\right| \leq C_{7}\left(1+\left|w^{\prime}\right|^{q-1}\right)\left|w^{\prime}\right|,
$$

so that

$$
\mathrm{T} 4 \leq \underbrace{2 C_{7} \varepsilon E^{\frac{p}{2}}\left|w^{\prime}\right|^{q}}_{\mathrm{T} 5}+\underbrace{2 C_{7} \varepsilon E^{\frac{p}{2}}\left|w^{\prime}\right|}_{\mathrm{T} 6} .
$$

Applying to the term T5 the same decomposition procedure as in the proof of Theorem 1.3 , we obtain, similarly to (1.29), the inequality

$$
\mathrm{T} 5 \leq C_{8}\left(\varepsilon \varepsilon^{q / 2} E(0)^{\frac{p q-1}{4}} E^{\frac{p+1}{2}}+\varepsilon^{1 / 2} E(0)^{\frac{p q-1}{4 q}}\left|w^{\prime}\right|^{q+1}\right) .
$$


Using Young's inequality with exponents $(p+1) / p$ and $p+1$ in the term T6, we obtain

$$
\mathrm{T} 6 \leq \frac{\varepsilon}{2} E^{\frac{p+1}{2}}+C_{9} \varepsilon\left|w^{\prime}\right|^{p+1} .
$$

By putting inequalities (1.17), (1.20), (1.34), (1.35), (1.36) together, rearranging the terms and using (1.32), it follows that

$$
\begin{aligned}
\phi^{\prime} & \leq \varepsilon\left(-\frac{1}{2}+C_{8} \varepsilon^{q / 2} E(0)^{\frac{p q-1}{4}}\right) E^{\frac{p+1}{2}}+\left(-c+\left(C_{4}+C_{9}\right) \varepsilon\right)\left|w^{\prime}\right|^{p+1} \\
& +\left(-c+C_{8} \varepsilon^{1 / 2} E(0)^{\frac{p q-1}{4 q}}\right)\left|w^{\prime}\right|^{q+1} .
\end{aligned}
$$

Now, if we take in formula (1.37)

$$
\varepsilon \leq \varepsilon_{1}:=\frac{C_{10}}{1+E(0)^{\frac{p q-1}{2 q}}} \leq \varepsilon_{0}
$$

(with $\varepsilon_{0}$ as defined by (1.19)), we find

$$
\phi^{\prime} \leq-\frac{\varepsilon}{4} E^{\frac{p+1}{2}} \leq-C_{11} \varepsilon \phi^{\frac{p+1}{2}} .
$$

Integrating this differential inequality yields

$$
E(t) \leq 2 \phi(t) \leq\left[\frac{C_{12}}{\phi(0)^{\frac{p-1}{2}}+\varepsilon t}\right]^{\frac{2}{p-1}}
$$

Choosing $\varepsilon=\varepsilon_{1}$, we finally obtain

$$
E(t) \leq C\left[1+E(0)^{\frac{p q-1}{q(p-1)}}\right] t^{-\frac{2}{p-1}}
$$

REMARK 1.2. It is easy to check that the constants $C$ in inequalities (1.11), (1.12), (1.26), and (1.33) depend on $h^{*}$ in a bounded manner. In fact, by resuming the proof of these results with a slightly more accurate use of (1.22), one could easily obtain explicit estimates on this dependence.

2. A stabilization theorem for a non-constant linear damping. In view of proving the exponential stability of the ODE (0.5) when $h$ is periodic, we here generalize a result of A. Haraux [7] on the stabilization of linearly damped abstract evolution equations of the form

$$
y^{\prime \prime}+A y+B y^{\prime}=0 .
$$

This "observability" result asserts that the zero solution is exponentially stable provided that the energy of the solutions to the undamped equation $\phi^{\prime \prime}+A \phi=0$ can be controlled by (or "observed through") the operator $B^{1 / 2}$ on a sufficiently long time interval.

We here extend this property to the case of non-constant linear damping. This property will be useful in section 3, for, when writing the difference of two solutions of (0.5), the nonlinear dissipation term will transform into a non-constant linear term.

In all of this section, $H$ denotes a real Hilbert space, $A: H \rightarrow H$ a linear (possibly unbounded) operator such that $A=A^{*} \geq 0, A$ coercive, $V=D\left(A^{1 / 2}\right), E=V \times H$. We also define a map $B \in L^{\infty}\left(\mathbf{R}^{+} ; \mathcal{L}(H)\right)$, such that $\forall t \in \mathbf{R}^{+}, B(t)=B^{*}(t) \geq 0$ is a bounded linear operator and $M=\sup _{t \geq 0}\|B(t)\|_{\mathcal{L}(H)}<+\infty$. 
We consider the following two equations:

$$
\begin{gathered}
y^{\prime \prime}+A y+B(t) y^{\prime}=0, \quad t \geq 0, \\
\phi^{\prime \prime}+A \phi=0, \quad t \geq 0 .
\end{gathered}
$$

We denote by $|u|$ the norm in $H$ and by $\|v\|$ the norm in $D\left(A^{1 / 2}\right)$. Let us set

$$
E_{y}(t)=\frac{1}{2}\left(\|y\|^{2}+\left|y^{\prime}\right|^{2}\right)(t) \text {. }
$$

We have the following theorem:

Theorem 2.1. Assume there exists some $T_{0} \geq 0, \tau, C>0$, such that for any solution $\phi$ of (2.2) we have

$$
\forall t \geq T_{0}, \quad\|\phi(t)\|^{2}+\left|\phi^{\prime}(t)\right|^{2} \leq C \int_{t}^{t+\tau}\left|B^{1 / 2}(s) \phi^{\prime}(s)\right|^{2} d s .
$$

Then there exists some $C_{1}, \delta>0$ such that for any $y$ solution of (2.1) we get

$$
\forall t \geq 0, \quad E_{y}(t) \leq C_{1} E_{y}(0) e^{\delta T_{0}} e^{-\delta t} .
$$

Moreover, $C_{1}$ and $\delta$ only depend on $\tau, C$, and $M$.

Proof. Take $t_{0} \geq 0,\left(y^{0}, y^{1}\right) \in E$, and $y$ the solution of $(2.1)$ such that

$$
y\left(t_{0}\right)=y^{0} \quad \text { and } \quad y^{\prime}\left(t_{0}\right)=y^{1} .
$$

The function $E:=E_{y}$ is nonincreasing and we have

$$
\int_{t_{0}}^{t_{0}+\tau}\left|B^{1 / 2}(s) y^{\prime}(s)\right|^{2} d s=E\left(t_{0}\right)-E\left(t_{0}+\tau\right) \leq E\left(t_{0}\right)
$$

Let $v$ be the solution of

$$
v^{\prime \prime}+A v=0, \quad t \in \mathbf{R}, \quad v\left(t_{0}\right)=y^{0}, \quad v^{\prime}\left(t_{0}\right)=y^{1} .
$$

Setting $w=y-v$, we obtain successively for $t_{0} \leq t \leq t_{0}+\tau$ :

$$
\begin{gathered}
E_{w}(T)=-\int_{t_{0}}^{t}\left(B(s) y^{\prime}(s), w^{\prime}(s)\right) d s \leq 2^{1 / 2} \int_{t_{0}}^{t}\left|B(s) y^{\prime}(s)\right| E_{w}^{1 / 2}(s) d s=: G(t), \\
G^{\prime}(t) \leq 2^{1 / 2}\left|B(t) y^{\prime}(t)\right| G^{1 / 2}(t) \leq(2 M)^{1 / 2}\left|B^{1 / 2}(t) y^{\prime}(t)\right| G^{1 / 2}(t),
\end{gathered}
$$

and integrating this differential inequality,

$$
G^{1 / 2}(t) \leq\left(\frac{M}{2}\right)^{1 / 2} \int_{t_{0}}^{t}\left|B^{1 / 2}(s) y^{\prime}(s)\right| d s \leq\left(\frac{M \tau}{2}\right)^{1 / 2}\left[\int_{t_{0}}^{t_{0}+\tau}\left|B^{1 / 2}(s) y^{\prime}(s)\right|^{2} d s\right]^{1 / 2} .
$$

Hence, by (2.5):

$$
\forall t \in\left[t_{0}, t_{0}+\tau\right], \quad E_{w}(t) \leq \frac{M \tau}{2}\left(E\left(t_{0}\right)-E\left(t_{0}+\tau\right)\right) .
$$

From (2.5) and (2.6), it follows that

$$
\begin{aligned}
\int_{t_{0}}^{t_{0}+\tau}\left|B^{1 / 2}(s) v^{\prime}(s)\right|^{2} d s & \leq 2 \int_{t_{0}}^{t_{0}+\tau}\left\{\left|B^{1 / 2}(s) w^{\prime}(s)\right|^{2}+\left|B^{1 / 2}(s) y^{\prime}(s)\right|^{2}\right\} d s \\
& \leq 2\left((M \tau)^{2}+1\right)\left(E\left(t_{0}\right)-E\left(t_{0}+\tau\right)\right) .
\end{aligned}
$$


Setting $K=C\left(1+(M \tau)^{2}\right)$, we deduce from (2.3) that

$$
E\left(t_{0}+\tau\right) \leq E\left(t_{0}\right)=E_{v}\left(t_{0}\right) \leq K\left(E\left(t_{0}\right)-E\left(t_{0}+\tau\right)\right) .
$$

Hence

$$
\forall t \geq T_{0}, \quad E(t+\tau) \leq \frac{K}{K+1} E(t)
$$

and, with $\rho=K /(K+1)$,

$$
\forall t \geq T_{0}, \quad E(t) \leq E\left(T_{0}+\tau\left[\frac{t-T_{0}}{\tau}\right]\right) \leq E\left(T_{0}\right) \rho^{\left[\frac{t-T_{0}}{\tau}\right]} \leq E(0) \rho^{\frac{t-T_{0}}{\tau}-1} .
$$

This last inequality being also valid on $\left[0, T_{0}\right]$, we finally get

$$
\forall t \geq 0, \quad E(t) \leq C_{1} E(0) e^{\delta T_{0}} e^{-\delta t},
$$

with $C_{1}=1+1 / \rho$ and $\delta=(1 / \tau) \log (1+1 / \rho)$ : the result is proved.

3. Exponential stability for the ordinary differential equation with periodic forcing. In this section, we turn our efforts to the ODE:

$$
u^{\prime \prime}+u+g\left(u^{\prime}\right)=h(t), \quad t \in \mathbf{R}^{+} .
$$

We are first proving the simple stability of the solutions of $(0.5)$ under minimal hypotheses (Theorem 3.1). Then supposing $h$ periodic, by applying this result together with the stabilization Theorem 2.1, we prove the exponential stability of the periodic solution whenever it exists (Theorem 3.3). In order to do so, we only require a very weak condition on $g$, that reduces to $g \not \equiv 0$ in the neighbourhood of 0 when $g$ is $C^{1}$. In the case when $g$ fulfills the global growth conditions of section 1 , we can take advantage of the general polynomial decay estimates previously established, and we finally become able to estimate the constants involved in the exponential stability (Theorem 3.4).

Theorem 3.1. Let us suppose $h: \mathbf{R} \rightarrow \mathbf{R}$ bounded, uniformly continuous, $g: \mathbf{R} \rightarrow \mathbf{R}$ continuous nondecreasing with $g(0)=0$, and satisfying

$$
g \not \equiv 0 \text { on a neighbourhood of } 0 .
$$

If $h$ is non-resonant, then any solution $v$ of $(0.5)$ satisfies

$$
\lim _{t \rightarrow+\infty}|u(t)-v(t)|+\left|u^{\prime}(t)-v^{\prime}(t)\right|=0 .
$$

Proof. We have

$$
E_{w}^{\prime}=-\left(g\left(u^{\prime}\right)-g\left(v^{\prime}\right)\right)\left(u^{\prime}-v^{\prime}\right) \leq 0
$$

Hence $\lim _{t \rightarrow+\infty} E_{w}(t)=l \geq 0$. As a consequence of the (locally compact) Ascoli's Theorem, we deduce that there exists an increasing map $\phi: \mathbf{N} \rightarrow \mathbf{N}$, and some functions $\bar{u}, \bar{v}, \bar{h}$, such that

$$
\begin{array}{ll}
u_{n}:=u(\cdot+\phi(n)) \underset{n \rightarrow \infty}{\longrightarrow} \bar{u} & \text { in } C_{b}^{2}(\mathbf{R}), \\
v_{n}:=v(\cdot+\phi(n)) \underset{n \rightarrow \infty}{\longrightarrow} \bar{v} & \text { in } C_{b}^{2}(\mathbf{R}),
\end{array}
$$


and

$$
h_{n}:=h(\cdot+\phi(n)) \underset{n \rightarrow \infty}{\longrightarrow} \bar{h} \quad \text { in } C_{b}^{0}(\mathbf{R})
$$

We then have

$$
\begin{aligned}
\bar{u}^{\prime \prime}+\bar{u}+g\left(\bar{u}^{\prime}\right) & =\bar{h}, \\
\bar{v}^{\prime \prime}+\bar{v}+g\left(\bar{v}^{\prime}\right) & =\bar{h},
\end{aligned}
$$

so that, setting $\bar{w}=\bar{u}-\bar{v}$, we get

$$
\bar{w}^{\prime \prime}+\bar{w}+g\left(\bar{u}^{\prime}\right)-g\left(\bar{v}^{\prime}\right)=0 .
$$

But for all $t \in \mathbf{R}, \lim _{n \rightarrow+\infty} E_{w}(t+\phi(n))=l$; hence $E_{\bar{w}}^{\prime} \equiv 0$ and $g\left(\bar{u}^{\prime}\right)=g\left(\bar{v}^{\prime}\right)$. Therefore $\bar{w}^{\prime \prime}+\bar{w}=0$ and $\bar{w}=\rho \cos (t+\alpha)$. Supposing that $\bar{w} \not \equiv 0$ and up to translating, we can write

$$
g\left(\bar{u}^{\prime}\right)=g\left(\bar{u}^{\prime}+\rho \cos t\right) \text { and } \rho \neq 0 .
$$

We now rely on the following:

LEMMA 3.2. Suppose $g$ as in the hypotheses of Theorem 3.1, and $\bar{u}^{\prime}$ satisfies (3.2). Then $g\left(\bar{u}^{\prime}\right)$ is constant on $\mathbf{R}$.

Proof of Lemma 3.2. Let $A=\left(g\left(\bar{u}^{\prime}\right)\right)\langle\mathbf{R}\rangle$. By (3.2) and the nondecreasing character of $g$, we deduce that for any $t$ in $\mathbf{R} \backslash(\pi / 2+\pi \mathbf{Z}), g$ is constant on the closed interval of bounds $\bar{u}^{\prime}(t)$ and $\bar{u}^{\prime}(t)+\rho \cos t$. Therefore, it is possible to write $A$ as

$$
A=\left(\bigcup_{\substack{k \in \mathbf{Z} \\ p \in \mathbf{N}}} A_{k}^{p}\right) \cup\left(g\left(\bar{u}^{\prime}\right)\right)\langle\pi / 2+2 \pi \mathbf{Z}\rangle,
$$

with

$$
\begin{aligned}
A_{k}^{p}=\left\{x \in A \mid \exists J_{x}\right. \text { closed interval } & \subset[\pi / 2+k \pi, \pi / 2+(k+1) \pi], \\
& \text { such that } \left.g\left(\bar{u}^{\prime}\right)=x \text { on } J_{x} \text { and }\left|J_{x}\right| \geq 1 /(p+1)\right\} .
\end{aligned}
$$

If we then consider $x_{1}, \ldots, x_{n}$ some distinct points of $A_{k}^{p}$, the corresponding intervals $J_{x_{1}}, \ldots, J_{x_{n}}$ are disjoint, so that $n /(p+1) \leq \pi$ and $A_{k}^{p}$ is finite. Therefore $A$ is countable and connected (continuous range of $\mathbf{R}$ ), and hence a single point, which proves the claim.

Proof of Theorem 3.1 (continued). By Lemma 3.2, we have

$$
g\left(\bar{u}^{\prime}\right)=g\left(\bar{u}^{\prime}+\rho \cos t\right)=C
$$

and since, by (3.1), $g$ is non-constant on any open interval containing 0 , we can suppose for instance:

$$
\begin{gathered}
\bar{u}^{\prime} \geq 0, \\
\bar{u}^{\prime}+\rho \cos t \geq 0 .
\end{gathered}
$$

On the one hand, by (3.3) and the boundedness of $\bar{u}$ :

$$
\exists l_{1} \in \mathbf{R}, \quad \lim _{t \rightarrow+\infty} \bar{u}(t)=l_{1},
$$


and on the other hand, integrating (3.4) yields

$$
\forall k \in \mathbf{Z}, \quad \int_{\pi / 2+2 k \pi}^{\pi+2 k \pi} \bar{u}^{\prime}(t) d t+\rho \int_{\pi / 2+2 k \pi}^{\pi+2 k \pi} \cos t d t \geq 0,
$$

that is, $\rho \leq \bar{u}(\pi+2 k \pi)-\bar{u}(\pi / 2+2 k \pi)$ and, by letting $t \rightarrow+\infty, \rho \leq 0$. Similarly, by integrating (3.4) over $[\pi+2 k \pi, 3 \pi / 2+2 k \pi]$, we find $\rho \geq 0$, hence $\rho=0$ : contradiction. We finally conclude that $\bar{w} \equiv 0$ and $\lim _{t \rightarrow+\infty} w(t)=\lim _{t \rightarrow+\infty} w^{\prime}(t)=0$.

We thank A. Haraux for having suggested to us an important simplification in the proof of Theorem 3.1 .

REMARK 3.1. Theorem 3.1 is in fact optimal. Indeed, if condition (3.1) is not satisfied, there is some $\varepsilon>0$ such that $g=0$ on $[-\varepsilon,+\varepsilon]$ and Eq. (0.5) bears all the (unstable) solutions $u(t)=A \cos t$ for $|A| \leq \varepsilon$.

In the case when a periodic solution exists, Theorem 3.1 , together with the stabilization Theorem 2.1, allow us to prove the exponential stability of the periodic solution under a single condition on the increase rate of $g$ near 0 .

Theorem 3.3. Let us suppose $h: \mathbf{R} \rightarrow \mathbf{R}, T$-periodic, continuous, non-constant, $g: \mathbf{R} \rightarrow$ $\mathbf{R}$ locally Lipschitz continuous, nondecreasing with $g(0)=0$, and satisfying

$$
\forall \varepsilon>0, \exists \eta>0, \exists \alpha, \beta \in[-\varepsilon, \varepsilon](\alpha<\beta), \quad \alpha \leq x<y \leq \beta \Rightarrow \frac{g(x)-g(y)}{x-y} \geq \eta .
$$

If Eq. (0.5) admits a periodic solution $u$, then it is unique and there exists a constant $\delta>0$ such that for any solution $v$ of $(0.5)$ :

$$
\exists M\left(v(0), v^{\prime}(0)\right)>0, \forall t \geq 0, \quad E_{u-v}(t) \leq M\left(v(0), v^{\prime}(0)\right) e^{-\delta t} .
$$

Proof. The uniqueness is clear. Let $v$ be another solution of $(0.5)$ and $w=u-v$. The function $w$ satisfies

$$
w^{\prime \prime}+w+a(t) w^{\prime}=0
$$

with

$$
a(t)= \begin{cases}\frac{g\left(u^{\prime}\right)-g\left(v^{\prime}\right)}{u^{\prime}-v^{\prime}} & \text { if } u^{\prime}(t)=v^{\prime}(t) \\ 1 & \text { otherwise. }\end{cases}
$$

According to Theorem 2.1, we just need to prove the existence of $T_{0} \geq 0, \tau, C>0$, such that for any $\phi$ solution of $\phi^{\prime \prime}+\phi=0$, we have

$$
\forall t \geq T_{0}, \quad \phi^{2}(t)+\phi^{\prime 2}(t) \leq C \int_{t}^{t+\tau} a(s) \phi^{\prime 2}(s) d s .
$$

Since $u$ (like $h$ ) is non-constant and periodic, there exist some $a, b>0$ such that $u^{\prime}\langle\mathbf{R}\rangle=$ $[-a, b]$ and thanks to hypothesis (3.5), we can find $c \in \mathbf{R}, \beta, \eta>0$, such that $J:=$ $[c-\beta, c+\beta] \subset[a, b]$ and

$$
c-\beta \leq x<y \leq c+\beta \Rightarrow \frac{g(x)-g(y)}{x-y} \geq \eta .
$$

We can then choose $t_{1} \in[0, T]$ with $u^{\prime}\left(t_{1}\right)=c$ and $\gamma \in(0, T]$ such that

$$
\forall t \in\left[t_{1}, t_{1}+\gamma\right], \quad\left|u^{\prime}(t)-c\right| \leq \beta / 2
$$


(note that $c, \beta, \eta, t_{1}$, and $\gamma$ only depend on $g$ and $h$ ). Besides, by Theorem 3.1 , we know that there exists some $T_{0} \geq 0$, such that

$$
\forall t \geq T_{0}, \quad\left|u^{\prime}(t)-v^{\prime}(t)\right| \leq \beta / 2
$$

$\left(T_{0}\right.$ now depends on $v(0)$ and $\left.v^{\prime}(0)\right)$. Therefore, we obtain

$$
\forall n \in \mathbf{N} \cap\left[T_{0} / T,+\infty\right), \forall t \in\left[n T+t_{1}, n T+t_{1}+\gamma\right], \quad a(t) \geq \eta .
$$

On the other hand, it is clear that

$$
\inf _{\substack{\alpha \in \mathbf{R} \\ s \geq 0}} \int_{s}^{s+\gamma} \sin ^{2}(\sigma+\alpha) d \sigma=\nu>0
$$

(with $\nu$ only depending on $g$ and $h$ through $\gamma$ ). Combining (3.10) and (3.11) yields

$$
\forall t \geq T_{0}, \quad \int_{t}^{t+2 T} a(\sigma) \sin ^{2}(\sigma+\alpha) d \sigma \geq \eta \nu,
$$

which immediately gives (3.8) and, by application of Theorem 2.1,

$$
\forall t \geq 0, \quad E_{w}(t) \leq C_{1} E_{w}(0) e^{\delta T_{0}} e^{-\delta t}
$$

with $C_{1}$ and $\delta$ depending only on $g$ and $h$ through the values of $\eta, \nu$, and $T$.

REMARK 3.2. When $g$ is of class $C^{1}$, it is easily seen that condition (3.5) is in fact equivalent to (3.1) (i.e., $g \not \equiv 0$ in the neighbourhood of 0 ), which is a necessary condition for any type of stability (cf. Remark 3.1). Exponential stability thus appears as a quite general phenomenon for Eq. (0.5).

REMARK 3.3. Concerning these results, it is worth noticing that the exponential stability property for equations of type (0.1) is generally obtained for only strongly monotone dissipative terms $g$, i.e., whose increase rate remains at least equal to some $k>0$ (cf. [6]). For this reason, the result of section 3 seems rather new and it would be interesting to extend it to the wave equation. Unfortunately, the observability method does not seem likely to work here, by lack of an $L^{\infty}$ estimate on the velocity $u^{\prime}$. In another direction, let us recall that the optimality of the (polynomial) time decay for $(0.3)$ with $h \equiv 0$ remains an open question.

The next result gives an estimate on the way the constant $M$ in (3.6) depends on $v(0)$ and $v^{\prime}(0)$, when $g$ satisfies the global polynomial growth conditions of section 1 .

TheOREM 3.4. Let us assume $h$ as in Theorem 3.3 and suppose that there exist some $p$, $q$, with $1<p \leq q$ and some constants $c, K>0$ such that

$$
\begin{aligned}
& \forall x, y \in \mathbf{R}, \quad c\left(|x-y|^{p+1}+|x-y|^{q+1}\right) \leq(g(x)-g(y))(x-y), \\
& \forall x, y \in \mathbf{R}, \quad|g(x)-g(y)| \leq K\left[1+(g(x) x+g(y) y)^{\frac{q-1}{q+1}}\right]|x-y| .
\end{aligned}
$$

Then, whenever (0.5) admits a periodic solution $u$, it is unique and any solution $v$ of (0.5) satisfies

$$
\forall t \geq 0, \quad E_{u-v}(t) \leq F\left(E_{u-v}(0)\right) e^{-\delta t}
$$


with

$$
F(X)=C X \exp \left[\varepsilon X^{\frac{p q-1}{2 q}}\right]
$$

and $C, \varepsilon, \delta>0$ only depending on $g$ and $h$.

Proof. Let $u$ be the periodic solution of (0.5) and $v$ another solution, $w=u-v$. From the proof of Theorem 3.3, we know that

$$
\forall t \geq 0, \quad E_{w}(t) \leq C_{1} E_{w}(0) e^{\delta T_{0}} e^{-\delta t},
$$

if $T_{0}$ satisfies

$$
\forall t \geq T_{0}, \quad\left|u^{\prime}(t)-v^{\prime}(t)\right| \leq \beta / 2
$$

with $C_{1}, \delta$, and $\beta>0$ only depending on $g$ and $h$. Using Theorem 1.4, we see that

$$
\forall t \geq 0, \quad\left|w^{\prime}(t)\right| \leq\left(2 E_{w}(t)\right)^{\frac{1}{2}} \leq(2 C)^{\frac{1}{2}}\left(1+E_{w}(0)^{\frac{p q-1}{2 q(p-1)}}\right) t^{\frac{-1}{p-1}}
$$

and therefore, (3.9) holds if we choose

$$
T_{0}=C_{1}\left(1+E_{w}(0)^{\frac{p q-1}{2 q}}\right) .
$$

The result follows immediately.

REMARK 3.4. Under the hypotheses of Theorem 3.4, the existence of the periodic solution can in fact be proved, as a consequence of Browder-Petryshyn's Theorem (see $[2])$, in the same way as in [6], Theorem IV.2.2.1.

\section{Solutions of the ODE on the real line and optimality of the constants.} In this section, we prove the existence of nontrivial global solutions on the real line for the ODE

$$
u^{\prime \prime}+f(u)=g\left(u^{\prime}\right)+h(t), \quad t \in \mathbf{R}
$$

for a rather wide class of functions $f, g$, and $h$ (subsection $a)$ ). In the case when $f(u)=u$, we prove that if $h$ is bounded and $g(y)$ essentially behaves like $|y|^{p-1} y(p>1)$ at infinity, these solutions behave also like a power, when $t \rightarrow+\infty$ (subsection $b)$ ). In order to prove the existence and behaviour results, we shall follow the methods we introduced in [9] and [10], thus generalizing the results previously obtained to the quasi-autonomous framework. The existence of such solutions will enable us to give estimates from below on the energy of the difference of two solutions of

$$
u^{\prime \prime}+u+g\left(u^{\prime}\right)=h(t), \quad t \geq 0
$$

and hence show the optimality of the constants involved in Theorems 3.1 and 3.4. Subsection c) is devoted to the general case when $h$ is bounded, featuring time-decay like a power of $t$ and polynomial growth of the constant for large energies. In subsection d), we will turn to the case of exponential time-decay (e.g., when $h$ is periodic), with exponential growth of the constant. 
a) Existence of global solutions. From now on, we suppose that $f, g: \mathbf{R} \rightarrow \mathbf{R}$ are some Lipschitz continuous functions and $h: \mathbf{R} \rightarrow \mathbf{R}$ is continuous. The main result is the following:

ThEOREM 4.1 (Existence). We assume there exists some constant $K>0$ such that

$$
\begin{gathered}
|f(x)| \leq\left(K^{2} / 4\right)|x|, \quad \text { for }|x| \text { large enough, } \\
\quad g(x) x \geq K x^{2}, \quad \text { for }|x| \text { large enough, } \\
\exists \lambda \in[0, K / 2),|h(t)| \leq e^{\lambda t}, \quad \text { for } t \text { large enough. }
\end{gathered}
$$

Then for every $t_{0} \in \mathbf{R}$ and for every $u_{0} \in \mathbf{R}$, there exists at least a solution of (4.1), global on $\mathbf{R}$, such that $u\left(t_{0}\right)=u_{0}$.

The proof relies on several intermediate steps; we first show by an energetic method that the maximal solutions are global near $-\infty$ (Proposition 4.2). Secondly, we prove that all the blow-up solutions are non-oscillatory (Proposition 4.3). We then establish that the maximal solutions have their differential tending to $\pm \infty$ whenever the initial data lie in certain areas of the phase plane (Proposition 4.4). The existence of a global solution for each value of $u\left(t_{0}\right)$ will then follow from the application of the theorem of continuity of the solutions in a critical range of values of $u^{\prime}\left(t_{0}\right)$ where both types of growing-up solutions cohabitate.

In the sequel, $T^{*}$ denotes the upper bound of the existence interval of a maximal solution.

Proposition 4.2. Let us assume that

$$
\begin{aligned}
& \exists K>0, \quad|f(x)| \leq K|x|, \quad \text { for }|x| \text { large enough. } \\
& \exists p>0, \exists k>0, \quad g(x) x \geq k|x|^{p+1}, \quad \text { for }|x| \text { large enough. }
\end{aligned}
$$

Then the existence interval $I$ of a maximal solution $u$ of Eq. (4.1) is of the form $\left(-\infty, T^{*}\right)$, $T^{*} \in \mathbf{R} \cup\{+\infty\}$.

Proof. Let us consider the backward equation

$$
v^{\prime \prime}+f(v)=g\left(-v^{\prime}\right)+h(-t)
$$

satisfied by $v(t):=u(-t)$, whose existence interval we denote by $J$. We call $F$ the primitive of $f$ that vanishes in 0 and we set $E(t)=v^{\prime 2}+2 F(v(t))$. We have

$$
E^{\prime}(t)=2 v^{\prime}\left(v^{\prime \prime}+f(v)\right)=2 g\left(-v^{\prime}\right) v^{\prime}+2 h(-t) v^{\prime} .
$$

From hypothesis (4.6),

$$
\exists k^{\prime}>0, \forall x \in \mathbf{R}, \quad g(x) x \geq k|x|^{p+1}-k^{\prime},
$$

and by Young's inequality with exponents $p+1$ and $(p+1) / p$, we get

$$
\forall t, x \in \mathbf{R}, \quad h(-t) v^{\prime} \leq k\left|v^{\prime}\right|^{p+1}+B|h(-t)|^{\frac{p+1}{p}},
$$

for some constant $B>0$. Hence

$$
E^{\prime}(t) \leq 2 B|h(-t)|^{\frac{p+1}{p}}+2 k^{\prime}
$$


Taking $t_{0} \in J$ and integrating inequality (4.7), we obtain for every $t \geq t_{0}$ with $t \in J$ :

$$
E(T) \leq \phi(t)
$$

with $\phi$ (continuous on $\mathbf{R}$ ) a primitive of the right-hand of (4.7). From hypothesis (4.5),

$$
\exists K^{\prime}>0, \forall x \in \mathbf{R}, \quad|F(x)| \leq(K / 2)|x|^{2}+K^{\prime},
$$

which yields

$$
v^{\prime 2} \leq E(t)+2|F(t)| \leq K v^{2}+2 K^{\prime}+\phi(t) .
$$

Then

$$
\left(v^{2}\right)^{\prime}=2 v v^{\prime} \leq v^{2}+v^{\prime 2} \leq(1+K) v^{2}+2 K^{\prime}+\phi(t),
$$

so that

$$
\left[v^{2} \exp (-(1+K) t]^{\prime} \leq\left(\phi(t)+2 K^{\prime}\right) \exp (-(1+K) t) .\right.
$$

By integration, it follows that $v$ (and therefore $v^{\prime}$ too by $(4.8)$ ) is bounded on $\left[t_{0},+\infty\right) \cap J$ by a function that is continuous on $\mathbf{R}$. Thus we conclude that $\left[t_{0},+\infty\right) \subset J$ and $I=\left(-\infty, T^{*}\right)$.

Proposition 4.3. We now suppose

$$
\begin{aligned}
& \exists K>0, \quad|f(x)| \leq K|x|, \quad \text { for }|x| \text { large enough, } \\
& \exists \alpha>0, \forall x \in \mathbf{R}^{+}, \quad g(x) \geq-\alpha \quad \text { and } \quad \forall x \in \mathbf{R}^{-}, \quad g(x) \leq \alpha .
\end{aligned}
$$

Then for any maximal solution $u$ of Eq. (4.1), if $T^{*}<+\infty$, we have

$$
\lim _{t \rightarrow T^{*}} u^{\prime}(t)=+\infty \quad(\text { resp. }-\infty) \text {. }
$$

Proof. Let us suppose $T^{*}<+\infty$. Then $u^{\prime}$ is unbounded on any neighbourhood of $T^{*}$ and we can for instance assume that

$$
\sup _{T^{*}-\varepsilon_{0} \leq t<T^{*}} u^{\prime}(t)=+\infty
$$

for some $\varepsilon_{0}>0$ (up to replacing $u$ by $-u, f$ by $x \mapsto-f(-x)$ and $g$ by $x \mapsto-g(-x)$ ). In addition, we may set

$$
M:=\min _{T^{*}-\varepsilon_{0} \leq t<T^{*}} h(t)>-\infty
$$

since $h$ is continuous.

Let us now assume that we do not have $\lim _{t \rightarrow T^{*}} u^{\prime}(t)=+\infty$. From (4.5),

$$
\exists K^{\prime}>0, \forall x \in \mathbf{R}, \quad f(x) \leq K|x|+K^{\prime}
$$

and for any $A$ large enough,

$$
\forall \delta>0, \exists t \in\left[T^{*}-\delta, T^{*}\right), \quad u^{\prime}(t) \leq A .
$$

Taking $\varepsilon>0$, with $\varepsilon \leq \varepsilon_{0}$, then

$$
\exists t_{1}=\min \left\{t \in\left[T^{*}-\varepsilon, T^{*}\right) \mid u^{\prime}(t) \geq 2 A\right\}
$$

and

$$
\exists t_{2}=\min \left\{t \in\left(t_{1}, T^{*}\right) \mid u^{\prime}(t) \leq A\right\} .
$$


Moreover, there is some $t_{3}$ such that

$$
u^{\prime}\left(t_{3}\right)=\max \left\{u^{\prime}(t) \mid t \in\left[T^{*}-\varepsilon, t_{2}\right]\right\} \geq 2 A .
$$

We then have $t_{1} \leq t_{3}<t_{2}$ and

$$
\forall t \in\left[t_{3}, t_{2}\right], \quad 0<A \leq u^{\prime}(t) \leq u^{\prime}\left(t_{3}\right) .
$$

By the mean-value theorem,

$$
\exists t_{4} \in\left(t_{3}, t_{2}\right), \quad u^{\prime \prime}\left(t_{4}\right)=\frac{u^{\prime}\left(t_{2}\right)-u^{\prime}\left(t_{3}\right)}{t_{2}-t_{3}} .
$$

On the one hand, since $u^{\prime}\left(t_{2}\right)=A$, we find

$$
f\left(u\left(t_{4}\right)\right)=g\left(u^{\prime}\left(t_{4}\right)\right)+h\left(t_{4}\right)-u^{\prime \prime}\left(t_{4}\right) \geq-\alpha+M+\frac{u^{\prime}\left(t_{3}\right)-A}{t_{2}-t_{3}}
$$

hence

$$
f\left(u\left(t_{4}\right)\right) \geq-\alpha+M+\frac{u^{\prime}\left(t_{3}\right)}{2 \varepsilon} .
$$

But on the other hand, by definition of $t_{3}$ :

$$
u\left(t_{4}\right)=u\left(T^{*}-\varepsilon\right)+\int_{T^{*}-\varepsilon}^{t_{4}} u^{\prime}(s) d s \leq u\left(T^{*}-\varepsilon\right)+\varepsilon u^{\prime}\left(t_{3}\right),
$$

so that

$$
\begin{aligned}
f\left(u\left(t_{4}\right)\right) & \leq K^{\prime}+K\left|u\left(T^{*}-\varepsilon\right)+\varepsilon u^{\prime}\left(t_{3}\right)\right| \\
& \leq K^{\prime}+K\left|u\left(T^{*}-\varepsilon\right)\right|+K \varepsilon u^{\prime}\left(t_{3}\right) .
\end{aligned}
$$

Combining inequalities (4.10) and (4.11) yields

$$
\left[\frac{1}{2 \varepsilon}-K \varepsilon\right] u^{\prime}\left(t_{3}\right) \leq K^{\prime}+K\left|u\left(T^{*}-\varepsilon\right)\right|+\alpha-M .
$$

Choosing $\varepsilon=\min \left(\varepsilon_{0}, 1 /(2 \sqrt{K})\right)$, this becomes

$$
\sqrt{K} A \leq \sqrt{K} u^{\prime}\left(t_{3}\right) / 2 \leq K^{\prime}+K\left|u\left(T^{*}-\varepsilon\right)\right|+\alpha-M .
$$

Since $K$ and $K^{\prime}$ are fixed, we can let $A$ tend to infinity and we obtain a contradiction.

Proposition 4.4. We assume that there exists a constant $K>0$ such that

$$
\begin{aligned}
& |f(x)| \leq\left(K^{2} / 4\right)|x|, \quad \text { for }|x| \text { large enough, } \\
& g(x) x \geq K x^{2}, \quad \text { for }|x| \text { large enough, } \\
& \exists \lambda \in[0, K / 2), \quad|h(t)| \leq e^{\lambda t}, \quad \text { for } t \text { large enough. }
\end{aligned}
$$

Then there exist some constants $\varepsilon, \gamma, \delta>0(\delta<\varepsilon)$ depending only on $f, g$, and $h$, and for any $\alpha \geq 0$ and $T_{0} \in \mathbf{R}$ there exists $C\left(\alpha, T_{0}\right)>0$ such that for any maximal solution $u$ of Eq. (4.1) (global or not for $t \rightarrow+\infty$ ), the following properties hold. then

a) If $\exists t_{0} \in I, t_{0} \geq T_{0}, u\left(t_{0}\right) \geq-\alpha, u^{\prime}\left(t_{0}\right) \geq \gamma$, and $u^{\prime}\left(t_{0}\right) \geq \varepsilon u\left(t_{0}\right)+e^{\delta t_{0}}+C\left(\alpha, T_{0}\right)$,

$$
\lim _{t \rightarrow T^{*}} u^{\prime}(t)=+\infty \quad \text { with } \forall t \geq t_{0}, u^{\prime}(t) \geq \gamma
$$


b) If $\exists t_{0} \in I, t_{0} \leq T_{0}, u\left(t_{0}\right) \leq \alpha, u^{\prime}\left(t_{0}\right) \leq-\gamma$, and $u^{\prime}\left(t_{0}\right) \leq \varepsilon u\left(t_{0}\right)-e^{\delta t_{0}}-C\left(\alpha, T_{0}\right)$, then

$$
\lim _{t \rightarrow T^{*}} u^{\prime}(t)=-\infty \quad \text { with } \quad \forall t \geq t_{0}, u^{\prime}(t) \leq-\gamma .
$$

Proof. We take $\gamma>0$ such that

$$
|x| \geq \gamma \Rightarrow\left(|f(x)| \leq\left(K^{2} / 4\right)|x| \text { and } g(x) x \geq K x^{2}\right) .
$$

Let $\alpha \geq 0$ and $T_{0} \in \mathbf{R}$. We set $\varepsilon=K / 2$ and $\delta=(\varepsilon+\lambda) / 2 \in(\lambda, \varepsilon)$. From (4.4), $h(t)+(\varepsilon-\delta) e^{\delta t} \geq(\varepsilon-\delta) e^{\delta t}-e^{\lambda t}$ for $t$ large enough and therefore tends to $+\infty$, as $t$ tends to $+\infty$. As a consequence,

$$
\exists B\left(T_{0}\right)>0, \forall t \geq T_{0}, \quad h(t)+(\varepsilon-\delta) e^{\delta t}+B\left(T_{0}\right) \geq 0 .
$$

Let us set

$$
\beta=\max (\alpha, \gamma), \quad M=\max _{x \in[-\beta,+\beta]}|f(x)|, \quad \text { and } \quad C\left(\alpha, T_{0}\right)=1+\frac{K \beta}{2}+\frac{2\left(M+B\left(T_{0}\right)\right)}{K},
$$

and consider the function

$$
\phi(t)=u^{\prime}-\varepsilon u-e^{\delta t}-C\left(\alpha, T_{0}\right)
$$

We first show the propagation of the positivity of $\phi$ along the solutions; more precisely, let us show that

$$
\left.\forall t \geq T_{0}, \quad\left(u(t) \geq-\alpha, u^{\prime}(t) \geq \gamma, \text { and } \phi(t) \geq 0\right) \Rightarrow\left(\phi^{\prime}(t)\right)>0 \text { and } u^{\prime \prime}(t)>0\right) .
$$

Let us assume $u \geq-\alpha, u^{\prime} \geq \gamma$, and $\phi \geq 0$ for some $t \geq T_{0}$. We then have

$$
\begin{aligned}
\phi^{\prime}(t) & =u^{\prime \prime}-\varepsilon u^{\prime}-\delta e^{\delta t} \\
& =g\left(u^{\prime}\right)-f(u)+h(t)-\varepsilon u^{\prime}-\delta e^{\delta t} \\
& \geq \varepsilon u^{\prime}-f(u)+h(t)-\delta e^{\delta t} \\
& \geq \varepsilon^{2} u+\varepsilon e^{\delta t}+\varepsilon C\left(\alpha, T_{0}\right)-f(u)+h(t)-\delta e^{\delta t}
\end{aligned}
$$

and finally by (4.13),

$$
\begin{aligned}
\phi^{\prime}(t) & \geq\left(\varepsilon^{2} u-f(u)\right)+\left(h(t)+(\varepsilon-\delta) e^{\delta t}+B\left(T_{0}\right)\right)+\varepsilon+\beta \varepsilon^{2}+M \\
& \geq\left(\varepsilon^{2} u-f(u)\right)+\varepsilon+\beta \varepsilon^{2}+M .
\end{aligned}
$$

At this point only two cases are possible:

- If $u(t) \geq \gamma$, then $f(u(t)) \leq \varepsilon^{2} u(t)$, so that $\phi^{\prime}(t) \geq \varepsilon>0$.

- If $-\alpha \leq u(t) \leq \gamma$, then $\phi^{\prime}(t) \geq \varepsilon^{2}(u(t)+\beta)+(M-f(u(t))+\varepsilon \geq \varepsilon>0$.

Moreover,

$$
\left(u^{\prime}(t) \geq \gamma \text { and } \phi^{\prime}(t) \geq 0\right) \Rightarrow u^{\prime \prime}(t) \geq \varepsilon u^{\prime}(t) \geq \varepsilon \gamma>0,
$$

so that (4.14) is proved.

Thus, if we have

$$
\exists t_{0} \in I, t_{0} \geq T_{0}, u\left(t_{0}\right) \geq-\alpha, u^{\prime}\left(t_{0}\right) \geq \gamma, \text { and } u^{\prime}\left(t_{0}\right) \geq \varepsilon u\left(t_{0}\right)+e^{\delta t_{0}}+C\left(\alpha, T_{0}\right),
$$

it follows that

$$
\forall t \geq t_{0}, \quad u^{\prime \prime}(t) \geq \varepsilon u^{\prime}(t)>0
$$


If $T^{*}<+\infty$, by Proposition 4.3, we then have obviously $\lim _{t \rightarrow T^{*}} u^{\prime}(t)=+\infty$.

If $T^{*}=+\infty$, an immediate integration of (4.15) yields the same result.

The symmetrical case b) is obtained by changing $u$ in $-u, f$ in $x \mapsto-f(-x)$, and $g$ in $x \mapsto-g(-x)$. The proof is now complete.

REMARK 4.1. If one replaces the hypothesis (4.4) in Proposition 4.4 by " $h$ is bounded on $\mathbf{R} "$, it is easily seen that the result still holds, when replacing $u^{\prime}\left(t_{0}\right) \leq \varepsilon u\left(t_{0}\right)-e^{\delta t_{0}}-$ $C\left(\alpha, T_{0}\right)$ in a) by $u^{\prime}\left(t_{0}\right) \leq \varepsilon u\left(t_{0}\right)-C\left(\alpha, T_{0}\right)$, and the similar change in $\left.\mathrm{b}\right)$.

Thanks to these preliminary results, we are now able to provide the proof of Theorem 4.1.

Proof of Theorem 4.1. Let $t_{0} \in \mathbf{R}$ and $u_{0} \in \mathbf{R}$ and assume that

There is no global solution such that $u\left(t_{0}\right)=u_{0}$.

Denote by $\mathcal{A}^{+}$(resp. $\mathcal{A}^{-}$) the set of values $u_{0}^{\prime}$ such that the maximal solution reaching $\left(u_{0}, u_{0}^{\prime}\right)$ at $t_{0}$ be non-global with $\lim _{t \rightarrow T^{*}} u^{\prime}(t)=+\infty\left(\right.$ resp. $\left.\lim _{t \rightarrow T^{*}} u^{\prime}(t)=-\infty\right)$.

The essential part of the argument then lies in the following:

Lemma 4.5. Under the assumption (4.16), the sets $\mathcal{A}^{+}$and $\mathcal{A}^{-}$are open.

Proof of Lemma 4.5. We take $u_{0}^{\prime} \in \mathcal{A}^{+}$, denote by $u$ the maximal solution such that $u\left(t_{0}\right)=u_{0}$ and $u^{\prime}\left(t_{0}\right)=u_{0}^{\prime}$, and set $\alpha=\left|u_{0}\right|+1 /(2 \varepsilon)$.

Let us first show that there exists a time $t_{1} \in\left(t_{0}, T^{*}\right)$ such that

$$
u^{\prime}\left(t_{1}\right) \geq \gamma+1 \quad \text { and } \quad u^{\prime}\left(t_{1}\right) \geq \varepsilon u\left(t_{1}\right)+e^{\delta t_{1}}+C\left(\alpha, t_{0}\right)+1 .
$$

First, $\lim _{t \rightarrow T^{*}} u^{\prime}(t)=+\infty$ implies that

$$
\exists T \in\left(t_{0}, T^{*}\right), \forall t \in\left[T, T^{*}\right), \quad u^{\prime}(t) \geq \gamma+1 .
$$

Now, if we had

$$
\forall t \in\left[T, T^{*}\right), u^{\prime}(t) \leq \varepsilon u(t)+e^{\delta t}+C\left(\alpha, t_{0}\right)+1,
$$

then we would have

$$
\forall t \in\left[T, T^{*}\right), \quad\left\{\left[u(t)+\frac{C\left(\alpha, t_{0}\right)+1}{\varepsilon}\right] e^{-\varepsilon t}+\frac{1}{\varepsilon-\delta} e^{-(\varepsilon-\delta) t}\right\}^{\prime} \leq 0,
$$

so that

$$
\forall t \in\left[T, T^{*}\right), \quad u(t) \leq\left[|u(T)|+\frac{C\left(\alpha, t_{0}\right)+1}{\varepsilon}+\frac{e^{\delta T}}{\varepsilon-\delta}\right] e^{\varepsilon(t-T)},
$$

which combined with (4.17), (4.18) would imply that $u$ be global.

Let us now apply the theorem of continuity of the solutions (see, e.g., [5]): there exists some $\eta>0$ such that if $v$ is a maximal solution that verifies

$$
\left|v\left(t_{0}\right)-u_{0}\right| \leq \eta \quad \text { and } \quad\left|v^{\prime}\left(t_{0}\right)-u_{0}^{\prime}\right| \leq \eta,
$$

then $v$ exists on $\left[t_{0}, t_{1}\right]$ and we have

$$
\left|v\left(t_{1}\right)-u\left(t_{1}\right)\right| \leq \frac{1}{2 \varepsilon} \quad \text { and } \quad\left|v^{\prime}\left(t_{1}\right)-u^{\prime}\left(t_{1}\right)\right| \leq 1 / 2 .
$$


But by Proposition 4.4, $u^{\prime} \geq \gamma>0$ on $\left[t_{0}, T^{*}\right)$; hence $u\left(t_{1}\right) \geq u\left(t_{0}\right)$. Thus, whenever $v\left(t_{0}\right)=u_{0}$ and $\left|v^{\prime}\left(t_{0}\right)-u_{0}^{\prime}\right| \leq \eta$, we obtain

$$
\begin{gathered}
v\left(t_{1}\right) \geq u\left(t_{1}\right)-\frac{1}{2 \varepsilon} \geq u\left(t_{0}\right)-\frac{1}{2 \varepsilon} \geq-\left(\left|u\left(t_{0}\right)\right|+\frac{1}{2 \varepsilon}\right)=-\alpha, \\
v^{\prime}\left(t_{1}\right) \geq u^{\prime}\left(t_{1}\right)+\left|v^{\prime}\left(t_{1}\right)-u^{\prime}\left(t_{1}\right)\right| \geq \gamma+1-1 / 2 \geq \gamma
\end{gathered}
$$

and

$$
\begin{aligned}
v^{\prime}\left(t_{1}\right) & -\varepsilon v\left(t_{1}\right)-e^{\delta t_{1}}-C\left(\alpha, t_{0}\right) \\
& \geq u^{\prime}\left(t_{1}\right)-\varepsilon u\left(t_{1}\right)-e^{\delta t_{1}}-C\left(\alpha, t_{0}\right)-\left|v^{\prime}\left(t_{1}\right)-u^{\prime}\left(t_{1}\right)\right|-\varepsilon\left|v\left(t_{1}\right)-u\left(t_{1}\right)\right| \\
& \geq 1-1 / 2-1 / 2=0 .
\end{aligned}
$$

Then as a consequence of Proposition 4.4, $v^{\prime}\left(t_{0}\right) \in \mathcal{A}^{+}$and $\mathcal{A}^{+}$is open. The same argument proves that $\mathcal{A}^{-}$is open and the Lemma follows.

Proof of Theorem 4.1 (continued). From (4.16) and Proposition 4.3, we know that

$$
\mathcal{A}^{+} \cup \mathcal{A}^{-}=\mathbf{R}
$$

and, by Proposition 4.4,

$$
\mathcal{A}^{+} \neq \emptyset \text { and } \mathcal{A}^{-} \neq \emptyset
$$

(we just need to take respectively $u_{0}^{\prime}=\max \left(\gamma, \varepsilon u_{0}+e^{\delta t_{0}}+C\left(\alpha, t_{0}\right)\right)$ and $u_{0}^{\prime}=\min (-\gamma$, $\left.\left.\varepsilon u_{0}-e^{\delta t_{0}}-C\left(\alpha, t_{0}\right)\right)\right)$. This contradicts the fact that $\mathbf{R}$ is connected. Thus, assumption (4.16) has to be discarded, and the proof of Theorem 4.1 is now complete.

As an immediate consequence, we have the following:

Corollary 4.6. If $f, g$, and $h$ satisfy

$$
\begin{aligned}
& \exists C>0, \quad|f(x)| \leq C|x|, \quad \text { for }|x| \text { large enough, } \\
& \lim _{|x| \rightarrow \infty} \frac{g(x)}{x}=+\infty, \\
& \exists C^{\prime}>0, \quad|h(t)| \leq e^{C^{\prime} t}, \quad \text { for } t \text { large enough, }
\end{aligned}
$$

then, for every $t_{0} \in \mathbf{R}$ and for every $u_{0} \in \mathbf{R}$, there exists at least a solution of (4.1), global on $\mathbf{R}$, such that $u\left(t_{0}\right)=u_{0}$.

Proof of Corollary 4.6. It suffices to apply Theorem 4.1 with $K>\max \left(2 \sqrt{C}, 2 C^{\prime}\right)$.

b) Asymptotic behaviour. As announced above, we consider in this subsection the particular case of the equation

$$
u^{\prime \prime}+u=g\left(u^{\prime}\right)+h(t), \quad t \in \mathbf{R} .
$$

The function $g: \mathbf{R} \rightarrow \mathbf{R}$ is locally Lipschitz continuous, $h: \mathbf{R} \rightarrow \mathbf{R}$ is continuous. Besides we shall always assume that

There exists a solution $u$ of (4.22) such that $u$ and $u^{\prime}$ are bounded on $\mathbf{R}$.

We know that this last assumption is verified, in particular, if $g$ and $h$ satisfy the hypotheses of Theorem 1.4. Indeed, in this case, by considering the solutions $u_{n}$ of $(0.5)$ on $[-n,+\infty)$ such that $u_{n}(0)=u_{n}^{\prime}(0)=0$, we can use the estimates obtained on the 
difference of two solutions to show that $\left(u_{n}\right)_{n}$ is a Cauchy sequence (cf. [6], Remark V.3.2.3).

The main result is the following:

TheOREM 4.7 (Asymptotic behaviour). Suppose

$$
\begin{aligned}
& \exists p>1, \exists B_{1}, B_{2}>0, \quad B_{1}|x|^{p} \leq|g(x)| \leq B_{2}|x|^{p}, \quad \text { for }|x| \text { large, } \\
& \exists \gamma \geq 0, \exists C>0, \forall x, y \in \mathbf{R}, \quad(g(x)-g(y))(x-y) \geq C|x-y|^{\gamma+1}, \\
& h \text { is bounded. }
\end{aligned}
$$

Then there exist some constants $K_{1}, K_{2}>0$ such that for any global solution $v \not \equiv u$ of (4.22), we have the estimate

$$
\left.K_{1} t^{\frac{1}{p-1}} \leq v^{\prime}(t) \text { (resp. }-v^{\prime}(t)\right) \leq K_{2} t^{\frac{1}{p-1}}, \quad \text { for } t \text { large enough. }
$$

In order to prove this, we first need to establish some preliminary results.

Proposition 4.8. Suppose

$$
\exists \gamma \geq 0, \exists C>0, \forall x, y \in \mathbf{R}, \quad(g(x)-g(y))(x-y) \geq C|x-y|^{\gamma+1} .
$$

Then any global solution $v \not \equiv u$ of (4.22) satisfies

$$
\lim _{t \rightarrow+\infty} E_{v}(t)=+\infty
$$

Proof of Proposition 4.8. Setting $w=u-v \not \equiv 0$, we have

$$
E_{w}^{\prime}(t)=\left(g\left(u^{\prime}\right)-g\left(v^{\prime}\right)\right) w^{\prime} \geq C\left|w^{\prime}\right|^{\gamma+1} \geq 0
$$

so that $\exists l \in \mathbf{R}^{+*} \cup\{+\infty\}, \lim _{t \rightarrow+\infty} E_{w}(t)=l$. Suppose $l$ is finite. From (4.28), we see that

$$
\int_{0}^{+\infty}\left|w^{\prime}\right|^{\gamma+1}(t) d t \text { is finite. }
$$

But on the other hand, $w, w^{\prime}, u, u^{\prime}$, and therefore $v, v^{\prime}$ are bounded on $\mathbf{R}^{+}$and so is $w^{\prime \prime}$. This implies that the function $\left|w^{\prime}\right|^{\gamma+1}$ is uniformly continuous on $\mathbf{R}^{+}$, which, combined with (4.29), yields

$$
\lim _{t \rightarrow+\infty} w^{\prime}(t)=0
$$

and then

$$
\lim _{t \rightarrow+\infty} w(t)=l^{\prime}= \pm \sqrt{2 l}
$$

From the boundedness of $u^{\prime}$, we then obtain that the right-hand side of the equation

$$
w^{\prime \prime}+w=g\left(u^{\prime}\right)-g\left(u^{\prime}+w^{\prime}\right)
$$

tends to zero at infinity, so that $\lim _{t \rightarrow+\infty} w^{\prime \prime}(t)=-l^{\prime} \neq 0$ : contradiction. We thus conclude that $l=+\infty$, and the result follows. 
Proposition 4.9. Suppose

$\exists \gamma \geq 0, \exists C>0, \forall x, y \in \mathbf{R}, \quad(g(x)-g(y))(x-y) \geq C|x-y|^{\gamma+1}$,

$h$ is bounded,

$g(x) x \geq 2 x^{2}, \quad$ for $|x|$ large enough.

Then for any global solution $v \not \equiv u$ of (4.22), we have

$$
\lim _{t \rightarrow+\infty} v(t)=\lim _{t \rightarrow+\infty} v^{\prime}(t)=+\infty \quad(\text { resp. }-\infty) .
$$

Proof of Proposition 4.9. Suppose that we do not have $\lim _{t \rightarrow+\infty}|v(t)|=+\infty$, i.e., there exists an increasing sequence $\left(t_{n}\right)_{n \in \mathbf{N}}$ tending to $+\infty$ such that

$$
\exists \alpha>0, \forall n \in \mathbf{N}, \quad\left|v\left(t_{n}\right)\right| \leq \alpha .
$$

By Proposition 4.8, we must have $\lim _{n \rightarrow \infty}\left|v^{\prime}\left(t_{n}\right)\right|=+\infty$ and, up to extracting a subsequence, we can suppose for instance that

$$
\lim _{n \rightarrow \infty} v^{\prime}\left(t_{n}\right)=+\infty
$$

In particular, with the notation of Proposition 4.4,

$$
\exists p \in \mathbf{N}, v\left(t_{p}\right) \geq-\alpha \quad \text { and } \quad v^{\prime}\left(t_{p}\right) \geq \max \left(\gamma, \varepsilon u\left(t_{p}\right)+C\left(\alpha, t_{0}\right)\right) .
$$

Then as a consequence of Proposition 4.4 and Remark 4.1, it follows that

$$
\lim _{t \rightarrow+\infty} v^{\prime}(t)=+\infty
$$

which is a contradiction. Therefore

$$
\lim _{t \rightarrow+\infty} v(t)=+\infty
$$

Take $A>0$. Since $g$ is continuous and $g$ is nonpositive on $\mathbf{R}^{-}$, there exists some $M>0$, such that

$$
y \leq A \Rightarrow g(y) \leq M .
$$

From (4.31), there is a time $T>0$ such that

$$
\forall t \geq T, \quad v(t) \geq M+\|h\|_{\infty}+1 .
$$

As a consequence, for any $t \geq T$, we get

$$
\begin{aligned}
v^{\prime}(t) \leq A & \Rightarrow v^{\prime \prime}(t)=-v(t)+g\left(v^{\prime}(t)\right)+h(t) \leq-1 \\
& \Rightarrow \forall s \geq t, \quad v^{\prime \prime}(s)<-1 \\
& \Rightarrow \lim _{t \rightarrow+\infty} v(t)=-\infty: \text { contradiction. }
\end{aligned}
$$

Therefore

$$
\forall t \geq T, \quad v^{\prime}(t)>A
$$

and the second part of the claim follows.

These preliminary results allow us to conclude: 
Proof of Proposition 4.7. From Proposition 4.9, we can for instance suppose

$$
\lim _{t \rightarrow+\infty} v(t)=\lim _{t \rightarrow+\infty} v^{\prime}(t)=+\infty
$$

and, for $t \geq T$ large enough,

$$
v, v^{\prime}>0 \text { and } 2\|h\|_{\infty} \leq B_{1} v^{\prime p} \leq g\left(v^{\prime}\right) \leq B_{2} v^{\prime p} .
$$

For every $t \geq T$, we have the following implications:

$$
\begin{aligned}
\alpha:=\frac{v}{v^{\prime p}} \geq 2 B_{2} & \Rightarrow \frac{v-h(t)}{g\left(v^{\prime}\right)} \geq \frac{v}{B_{2} v^{\prime p}}-\frac{|h(t)|}{B_{2} v^{\prime p}}>2-1=1 \\
& \Rightarrow v^{\prime \prime}<0 \\
& \Rightarrow \alpha^{\prime}=\frac{1}{v^{\prime p-1}}-\frac{p v v^{\prime \prime}}{v^{\prime p+1}}>0 .
\end{aligned}
$$

Hence, for $t_{0} \geq T$,

$$
\begin{aligned}
\alpha\left(t_{0}\right) \geq 2 B_{2} & \Rightarrow \forall t \geq t_{0}, \quad \alpha(t) \geq 2 B_{2} \\
& \Rightarrow \forall t \geq t_{0}, \quad v^{\prime \prime}(t)<0 .
\end{aligned}
$$

This last assumption would contradict $\lim _{t \rightarrow+\infty} v^{\prime}(t)=+\infty$. Thus

$$
\alpha(t) \leq 2 B_{2}, \quad \text { for } t \text { large enough. }
$$

On the other hand, take $\varepsilon>0$ such that $0<\varepsilon \leq B_{1} / 2$. For every $t \geq T$, we have, using (4.32):

$$
\begin{aligned}
\alpha \leq \varepsilon & \Rightarrow \frac{v-h(t)}{g\left(v^{\prime}\right)} \leq \frac{v}{B_{1} v^{\prime p}}+\frac{|h(t)|}{B_{1} v^{\prime p}} \leq \frac{1}{2}+\frac{1}{2}=1 \\
& \Rightarrow v^{\prime \prime} \geq 0 \\
& \Rightarrow \alpha^{\prime} \leq \frac{1}{v^{\prime p-1}} .
\end{aligned}
$$

But since $\lim _{t \rightarrow+\infty} v^{\prime}(t)=+\infty$, we can find some $T_{\varepsilon} \geq 0$, such that

$$
\forall t \geq T_{\varepsilon} \quad\left(\alpha(t) \leq \varepsilon \Rightarrow \alpha^{\prime}(t) \leq \varepsilon / 2\right) .
$$

Suppose now that $\alpha\left(t_{0}\right)<\varepsilon / 2$ for some $t_{0} \geq T_{\varepsilon}$. We have

$$
\forall t \in\left[t_{0}, t_{0}+1\right], \quad \alpha(t)<\varepsilon .
$$

(Otherwise, setting $t_{1}=\min \left\{t \in\left[t_{0}, t_{0}+1\right] \mid \alpha(t)=\varepsilon\right\}$, we would have $\alpha \leq \varepsilon$ and $\alpha^{\prime} \leq \varepsilon / 2$ on $\left[t_{0}, t_{1}\right]$. But then, there would be some $c \in\left[t_{0}, t_{1}\right]$ such that

$$
\alpha^{\prime}(c)=\frac{\alpha\left(t_{1}\right)-\alpha\left(t_{0}\right)}{t_{1}-t_{0}}>\frac{\varepsilon-\varepsilon / 2}{t_{1}-t_{0}} \geq \varepsilon / 2:
$$

contradiction.) As a consequence, on the interval $\left[t_{0}, t_{0}+1\right]$, it follows that

$$
\frac{v-h(t)}{g\left(v^{\prime}\right)} \leq \varepsilon / B_{1}
$$

so that

$$
\frac{v^{\prime \prime}}{v^{\prime p}} \geq \frac{B_{1} v^{\prime \prime}}{g\left(v^{\prime}\right)}=B_{1}\left(1-\frac{v-h(t)}{g\left(v^{\prime}\right)}\right) \geq B_{1}\left(1-\frac{\varepsilon}{B_{1}}\right)
$$


Choosing $\varepsilon=B_{1} / 2$ yields the differential inequality

$$
\forall t \in\left[t_{0}, t_{0}+1\right], \quad v^{\prime \prime}(t) \geq \frac{B_{1}}{2} v^{\prime p}(t) .
$$

After integration, there remains

$$
\frac{1}{v^{\prime p-1}\left(t_{0}+1\right)}-\frac{1}{v^{p-1}\left(t_{0}\right)} \leq-\frac{(p-1) B_{1}}{2}
$$

hence

$$
\frac{1}{v^{\prime p-1}\left(t_{0}\right)} \geq \frac{(p-1) B_{1}}{2}
$$

This last inequality contradicts $\lim _{t \rightarrow+\infty} v^{\prime}(t)=+\infty$, so that we must have

$$
\alpha(t) \geq \varepsilon / 2=\frac{B_{1}}{4} \quad \text { for } t \text { large enough. }
$$

Putting inequalities (4.33) and (4.34) together, one can see that the function

$$
\left[v^{\frac{p-1}{p}}\right]^{\prime}=\frac{(p-1) v^{\prime}}{p v^{1 / p}}
$$

remains bracketed between two positive constants as $t \rightarrow+\infty$, which gives the result (since these constants depend only on $B_{1}, B_{2}$, and $p$ ).

c) Optimality of the constants: the general case. We are here extending the method of [3] to the quasi-autonomous ODE framework. Taking advantage of the existence of global solutions to $(0.5)$ on the real line that grow up like a power near $-\infty$, we study the optimality of the constants $F\left(E_{u-v}(0)\right)$ involved in Theorem 1.4. The following result proves that (1.33) is optimal.

Proposition 4.10. We suppose $h: \mathbf{R} \rightarrow \mathbf{R}$ continuous, bounded, $g: \mathbf{R} \rightarrow \mathbf{R}$ locally Lipschitz continuous, satisfying:

$$
\begin{aligned}
& \exists q>1, \exists B_{1}, B_{2}>0, \quad B_{1}|x|^{q} \leq|g(x)| \leq B_{2}|x|^{q}, \quad \text { for }|x| \text { large, } \\
& \exists \gamma \geq 0, \exists C>0, \forall x, y \in \mathbf{R}, \quad(g(x)-g(y))(x-y) \geq C|x-y|^{\gamma+1}
\end{aligned}
$$

and we assume that Eq. (0.5) admits a solution $u$ such that $u$ and $u^{\prime}$ are bounded on $\mathbf{R}$. Suppose that an estimate of the form

$$
\forall t \geq 0, \quad E_{u-v}(t) \leq F\left(E_{u-v}(0)\right) t^{\frac{-2}{p-1}}
$$

holds for any solution $v$ of $(0.5)$, with $p>1, F: \mathbf{R}^{+} \rightarrow \mathbf{R}^{+}$nondecreasing, and that the estimate is valid for any $h$ of the same $L^{\infty}$ norm. Then the function $F$ necessarily satisfies

$$
F(X) \geq K X^{\frac{p q-1}{p(q-1)}}, \quad \text { as } X \rightarrow+\infty
$$

for some $K>0$.

Proof of Proposition 4.10. We know from Theorems 4.1 and 4.7 that Eq. (0.5) bears at least a global solution $v$ on the real line that satisfies:

$$
\exists A_{1}, A_{2}>0, \quad A_{1} s^{\frac{2 q}{q-1}} \leq E_{v}(-s) \leq A_{1} s^{\frac{2 q}{q-1}}, \quad \text { for } s \rightarrow+\infty .
$$


For all $\tau \geq 0$, we set $u_{\tau}=u(\cdot-\tau), v_{\tau}=v(\cdot-\tau)$, which are solutions of

$$
z^{\prime \prime}+z+g\left(z^{\prime}\right)=h_{\tau}(t):=h(t-\tau), \quad t \in \mathbf{R} .
$$

Since $\left\|h_{\tau}\right\|_{\infty}=\|h\|_{\infty}$, it follows, for any $s, \tau \geq 0$, that

$$
E_{u-v}(s-\tau)=E_{u_{\tau}-v_{\tau}}(s) \leq F\left(E_{u_{\tau}-v_{\tau}}(0)\right) s^{\frac{-2}{p-1}}=F\left(E_{u-v}(-\tau)\right) s^{\frac{-2}{p-1}} .
$$

From (4.37), and the boundedness of $\left(u, u^{\prime}\right)$, we deduce that

$$
A_{3} y^{\frac{2 q}{q-1}} \leq E_{u-v}(-y) \leq A_{4} y^{\frac{2 q}{q-1}}, \quad \text { for } y \rightarrow+\infty .
$$

Let us choose $\tau=2 s$. Using (4.38), (4.39) and the fact that $F$ is nondecreasing, we deduce, for $s$ large:

$$
A_{3} s^{\frac{2 q}{q-1}} \leq E_{u-v}(s) \leq F\left(A_{4} s^{\frac{2 q}{q-1}}\right) s^{\frac{-2}{p-1}} .
$$

Setting $X=A_{4} s^{\frac{2 q}{q-1}}$, the result follows easily.

REMARK 4.2. The optimality of the time rate of decay as a power of $t$ for $(0.5)$ is well known in the case $h \equiv 0$. This of course is no longer true in the general case, as indicated by Theorem 3.3. However, the result of Proposition 4.10 makes sense, since it only asserts the optimality of (1.33) among all possible estimates of the form (4.35).

REMARK 4.3. The conditions assumed on the function $F$ in Proposition 4.10 are justified by the fact that the available estimates satisfy such conditions (see Remark 1.2).

d) Optimality of the constants: the case of exponential decay. We first recall the wellknown fact that the time rate of decay for the difference of two (bounded) solutions of (0.5) cannot be faster than exponential. In fact, in the periodic case, under the hypotheses of Theorem 3.3, we now know that it is exactly exponential.

Proposition 4.11. Let us suppose $h: \mathbf{R} \rightarrow \mathbf{R}$ bounded, uniformly continuous, $g: \mathbf{R} \rightarrow$ $\mathbf{R}$ locally Lipschitz continuous nondecreasing with $g(0)=0$, and satisfying:

$$
g \not \equiv 0 \text { on a neighbourhood of } 0 .
$$

If Eq. (0.5) admits a solution $u_{0}$, such that $u_{0}$ and $u_{0}^{\prime}$ are bounded on $\mathbf{R}^{+}$, then for any distinct solutions $u$ and $v$ of $(0.5)$, we have

$$
\forall t \geq 0, \quad E_{u-v}(t) \geq C\left(u(0), u^{\prime}(0), v(0), v^{\prime}(0)\right) e^{-\varepsilon t}
$$

with $\varepsilon$ independent of $u, v$ and $C\left(u(0), u^{\prime}(0), v(0), v^{\prime}(0)\right)>0$.

Proof of Proposition 4.11. By Theorem 3.1, any solution is asymptotic to $u_{0}$ in the phase space when $t$ tends to $+\infty$. Therefore, there exists some $T_{1}>0$ depending on $u$ and $v$ such that

$$
\forall t \geq T_{1}, \quad\left|u^{\prime}(t)\right| \leq M \quad \text { and } \quad\left|v^{\prime}(t)\right| \leq M,
$$

with $M:=1+\left\|u_{0}^{\prime}\right\|_{L^{\infty}\left(\mathbf{R}^{+}\right)}$. Then setting $L:=\|g\|_{W^{1, \infty}([-M,+M])}$, we get

$$
\begin{aligned}
\forall t \geq T_{1}, \quad E_{u-v}^{\prime}(t) & =\left(g\left(u^{\prime}(t)\right)-g\left(v^{\prime}(t)\right)\right)\left(u^{\prime}(t)-v^{\prime}(t)\right) \\
& \geq-L\left(u^{\prime}(t)-v^{\prime}(t)\right)^{2} \geq-2 L E_{u-v}(t)
\end{aligned}
$$

and the result immediately follows by integration. 
Using the same method as in subsection c), we now establish a lower bound on the constant in the case of exponential time decay. As a consequence for the periodic framework, it appears that (3.14) in Theorem 3.4 is almost optimal, in the sense that the constant must necessarily behave exponentially for large initial energies. In precise terms, our result can be stated as:

Proposition 4.12. We suppose $h: \mathbf{R} \rightarrow \mathbf{R}$ continuous, $T$-periodic, $g: \mathbf{R} \rightarrow \mathbf{R}$ locally Lipschitz continuous, satisfying:

$$
\begin{aligned}
& \exists q>1, \exists B_{1}, B_{2}>0, \quad B_{1}|x|^{q} \leq|g(x)| \leq B_{2}|x|^{q}, \quad \text { for }|x| \text { large, } \\
& \exists \gamma \geq 0, \exists C>0, \forall x, y \in \mathbf{R}, \quad(g(x)-g(y))(x-y)] \geq C|x-y|^{\gamma+1},
\end{aligned}
$$

and we assume that Eq. (0.5) admits a $T$-periodic solution $u$. If an estimate of the form

$$
\forall t \geq 0, \quad E_{u-v}(t) \leq F\left(E_{u-v}(0)\right) e^{-\delta t}
$$

holds for any solution $v$ of $(0.5)$, with $F: \mathbf{R}^{+} \rightarrow \mathbf{R}^{+}$nondecreasing and $\delta>0$, then the function $F$ necessarily satisfies

$$
F(X) \geq K X \exp \left[\alpha X^{\frac{q-1}{2 q}}\right], \quad \text { as } X \rightarrow+\infty
$$

for some $K, \alpha>0$.

Proof of Proposition 4.12. We again consider a global solution $v$ of $(0.5)$ on the real line that satisfies (4.37). Setting $v_{n}=v(\cdot-n T)$ (which is a solution of $(0.5)$ since $h$ is periodic), it follows for any $n \in \mathbf{N}$ and $s \geq 0$, that

$$
E_{u-v}(s-n T)=E_{u-v_{n}}(s) \leq F\left(E_{u-v_{n}}(0)\right) e^{-\delta s}=F\left(E_{u-v}(-n T)\right) e^{-\delta s} .
$$

From (4.37), and the boundedness of $\left(u, u^{\prime}\right)$, it follows that

$$
A_{3} y^{\frac{2 q}{q-1}} \leq E_{u-v}(-y) \leq A_{4} y^{\frac{2 q}{q-1}}, \quad \text { for } y \rightarrow+\infty .
$$

We choose $n=[2 s / T]$, so that $s-T<n T-s \leq s$. Using (4.41), (4.42), and the fact that $F$ is nondecreasing, we deduce, for $s$ large:

$$
A_{3}(n T-s)^{\frac{2 q}{q-1}} \leq E_{u-v}(s-n T) \leq F\left(A_{4}(n T-s)^{\frac{2 q}{q-1}}\right) e^{-\delta s}
$$

and then

$$
A_{3}(s-T)^{\frac{2 q}{q-1}} \leq F\left(A_{4} s^{\frac{2 q}{q-1}}\right) e^{-\delta s} .
$$

Setting $X=A_{4} s^{\frac{2 q}{q-1}}$, the result follows.

REMARK 4.4. By comparing (3.14) and (4.40), one can see that the upper bound on $F$ tends to the lower one as $p$ goes to 1 .

\section{REFERENCES}

[1] N. Bogolioubov and Y. Mitropolsky, Asymptotic methods in the theory of nonlinear oscillations, Gordon and Breach Science Publ., New York, Hindustan Publ. Corp., Delhi, 1961

[2] F. Browder and W. Petryshyn, The solution by iteration of nonlinear functional equations in Banach spaces, Bull. Amer. Math. Soc. 72, 571-575 (1966)

[3] A. Carpio, Sharp estimates of the energy for the solutions of some dissipative second order evolution equations, Potential Analysis 1, 265-289 (1992)

[4] A. Carpio, Existence of global solutions to some nonlinear dissipative wave equations, J. Math. Pures Appl. 73, 471-488 (1994) 
[5] E. Coddington and N. Levinson, Ordinary Differential Equations, McGraw-Hill, 1955

[6] A. Haraux, Semi-linear hyperbolic problems in bounded domains, Mathematical Reports, Vol. 3, part 1, J. Dieudonné, Editor, Harwood Academic Publishers, Gordon and Breach, 1987

[7] A. Haraux, Une remarque sur la stabilisation de certains systèmes du deuxième ordre en temps, Portugaliae Mathematica 46, 3, 245-248 (1989)

[8] A. Haraux and E. Zuazua, Decay estimates for some nonlinear evolution equations, Arch. Rat. Mech. Anal. 100, 2, 191-206 (1988)

[9] Ph. Souplet, Étude des solutions globales de certaines équations différentielles ordinaires du second ordre non linéaires, Comptes-rendus de l'Académie des Sciences, t. 313, Série I, 365-370 (1991)

[10] $\mathrm{Ph}$. Souplet, Existence of exceptional growing-up solutions for a class of nonlinear second order ordinary differential equations, Asymptotic Analysis 11, 185-207 (1995)

[11] $\mathrm{Ph}$. Souplet, Propriétés globales de quelques équations d'évolution non linéaires du second ordre, Thèse, Université Pierre et Marie Curie, 1994 